\title{
Adaptive Control Allocation in the Presence of Actuator Failures
}

\author{
Yu Liu and Luis G. Crespo \\ National Institute of Aerospace, Hampton, VA 23666, USA \\ Correspondence should be addressed to Yu Liu, yl5c@virginia.edu
}

Received 3 November 2011; Revised 17 February 2012; Accepted 2 March 2012

Academic Editor: Yunjun Xu

Copyright ( $\odot 2012$ Y. Liu and L. G. Crespo. This is an open access article distributed under the Creative Commons Attribution License, which permits unrestricted use, distribution, and reproduction in any medium, provided the original work is properly cited.

\begin{abstract}
This paper proposes a control allocation framework where a feedback adaptive signal is designed for a group of redundant actuators and then it is adaptively allocated among all group members. In the adaptive control allocation structure, cooperative actuators are grouped and treated as an equivalent control effector. A state feedback adaptive control signal is designed for the equivalent effector and adaptively allocated to the member actuators. Two adaptive control allocation algorithms, guaranteeing closed-loop stability and asymptotic state tracking when partial and total loss of control effectiveness occur, are developed. Proper grouping of the actuators reduces the controller complexity without reducing their efficacy. The implementation and effectiveness of the strategies proposed is demonstrated in detail using several examples.
\end{abstract}

\section{Introduction}

Actuator redundancy is highly desirable for fault-tolerant control. This redundancy yields multiple ways to implement the forces and moments prescribed by the controller. However, this freedom creates the need for properly allocating the control inputs among all individual actuators. While multiple actuator configurations do generate the desired forces and moments, some of them may yield unintended outcomes, for example, the effect of some control surface deflections counteracts the effect of other ones. Redundancy management is the need for properly allocating the control inputs among functionally redundant actuators when some of them may not be fully functional.

The purpose of control allocation is to distribute the control signals to the available actuators such that the desired moments and forces are efficiently generated. Traditional control allocation methods include explicit ganging [1], daisy chaining [2], pseudinverse [3, 4], and error and control minimization [5-10]. Explicit ganging performs control allocation by finding a fixed relation between the desired control moments and forces and the designed control signals. Multiple actuators (e.g., two aileron surfaces) can be combined to generate the desired effects. Daisy chaining allocates inputs in a prioritized fashion. It utilizes the actuators in sequence to generate certain effect. If an actuator is unable to generate such an effect, say due to actuator saturation, the next actuator in the sequence will be used. The pseudoinverse approach, which accounts for input saturation and failure, performs control allocation by solving a linear optimization problem in real time. Error and control minimization is another common control allocation approach. This approach minimizes the error between the desired and generated control moments subject to control constraints. Several approaches can accommodate for actuator failure and saturation compensation, provided that the actuator failure or saturation has been identified. Thus one obvious drawback of these approaches is that they require a fault detection system. These systems, which are usually complex, require the characterization of several failure modes a priori. Furthermore, they may require solving optimization problems in real time; thus they can substantially increase the software and hardware demands of the flight control system.

Adaptive control, on the other hand, does not require knowing which controllers have failed nor the class or severity of the failure. This is due to its ability to change control parameters according to the existing flight condition. Due to parameter adaptation, it is also able to accommodate for parametric uncertainties in the vehicle 
dynamics. Substantial developments in adaptive control for actuator failures have been made in the last decade [11, 12]. Adaptive control's ability to seamlessly compensate for actuator failures requires for the system's built-in actuation redundancy to be sufficient. This is usually described as a rank condition on the input matrix $B[11,13]$. To take advantage of all redundancy available, one common approach in multivariable adaptive control is to generate a control signal for each control surface. Such an approach endows the controller the maximum degree of freedom to compensate for failure. When certain control surfaces are stuck or have reduced control effectiveness, the remaining control surfaces will adaptively cooperate until a new combination of inputs for the remaining control surfaces is found. This will occur automatically without knowing which surfaces have failed, or when such failures occur. Although adaptive control ensures closed-loop stability and tracking performance, it does not differentiate between control generation and control allocation, and the resulting actuation scheme may be unacceptable. For instance, separately designed control signals for multiple control surface segments may cancel each other's effects, for example, it has been observed that the steady-state deflection of both rudder segments for a direct adaptive control had opposite angles, resulting in a wings leveled flight with increased drag.

The lack of control allocation in the current direct adaptive control framework motivates this research effort. In this study, we aim at separating control generation from control allocation. In the adaptive control allocation framework, a key step is to combine redundant control surfaces similar to explicit ganging. For each group of combined actuators, we design an adaptive control signal, which is then allocated among group members by an adaptive gain. If no failure occurs, a nonadaptive control allocation scheme set in advance is enforced. In the presence of uncertainty of actuator failure, the adaptive flight controller modifies the allocation of input accordingly.

The structure of the adaptive control allocation framework is illustrated in Figure 1. This is a simple aircraft control example with the elevator controlling the pitch motion. The aircraft longitudinal state, denoted as $x(t)$, should track the state of a reference system for a given reference input $r(t)$. The elevator consists of four segments, namely, left outboard, left inboard, right outboard, and right inboard segments. For pitch control, they can be grouped together and considered as an equivalent elevator by adding the four columns of the input matrix. A "virtual" elevator signal $v_{0}$ is generated by the adaptive controller for the desired pitching maneuver. This elevator signal is then allocated by the adaptive allocation gains $\alpha_{i}(t), i=1, \ldots, 4$. The resulting elevator signals $v_{0 i}(t)=\alpha_{i}(t) v_{0}(t), i=1, \ldots, 4$ will be fed to the four elevator segments. The allocation gains can be updated on-line based on the knowledge of the nominal plant and $v_{0}$ to mitigate the uncertainties of actuator failures. Conversely, in a fixed allocation scheme $\alpha_{i}$ are constant, for example, $\alpha_{i}=1 / 4, i=1, \ldots, 4$.

One advantage of this adaptive control allocation structure is the ease in solving problems such as the counteracting actuation. To remedy this problem, the signs of the adaptive allocation gains $\alpha_{i}$ can be made the same, say, via the projection algorithm [14] so that the allocated control signals cannot go in opposite directions. Another advantage of this structure is the reduction of the controller complexity. For instance, let us consider a state feedback adaptive control design for an $n$-state system with $m$ controls. The number of controller parameters to be updated is $m \times n$. If the problem is solved by having all actuators in one group, there are $m+n$ adaptive parameters. For example, if we try to stabilize a 5 -state system with 3 controls, the total adaptive parameters using a direct model reference adaptive controller would be 15 . With a grouping of control inputs for which the system remains controllable (i.e., the system is controllable for the control input matrix associated with the grouping), the total number of adaptive parameters required is $8\left(1 \times 5\right.$ gain vector and $\left.3 \alpha^{\prime} \mathrm{s}\right)$. The larger the family of redundant actuators, the bigger the benefit. Another advantage of this approach is that the virtual control signal can be designed using a nonadaptive (fixed) state feedback gain, and failure compensation is achieved by only adapting the control allocation gains $\alpha$ 's. This implies that the proposed structure could be added to a conventionally designed state feedback controllers without having to use fault detection and isolation.

In this paper, we develop the mathematical foundation of this adaptive control allocation structure for a single group of actuators. Two adaptive control allocation algorithms are presented for both loss of effectiveness and constantmagnitude actuator failures. Technical issues such as design conditions, adaptive law designs, and stability analysis are addressed. The proposed schemes are shown to guarantee stability and asymptotic state tracking in the presence of unknown failures. Simulation-based examples are used to demonstrate the strategy proposed.

The paper is organized as follows. Section 2 presents the adaptive control allocation algorithm for loss of control effectiveness. This is followed by Section 3, where a scheme for constant-magnitude actuator failure compensation is developed in Section 3. Finally, a few concluding remarks close the paper.

\section{Adaptive Control Allocation Design for Loss of Effectiveness Failures}

\subsection{Problem Formulation}

\subsubsection{Plant. Consider the linear time-invariant (LTI) system}

$$
\dot{x}(t)=A x(t)+B u(t),
$$

where $x \in R^{n}$ and $u \in R^{m}$ are the system state and the control input. The matrices $A \in R^{n \times n}$ and $B \in R^{n \times m}$ are constant and known. The matrix $B$ is the control gain matrix for the group of actuators.

The control signal $u(t)$ can be expressed as

$$
u(t)=\Lambda v(t)
$$

where $v(t)$ is the allocated control signal (i.e., input to the actuator) and $\Lambda$ is a piecewise constant uncertain diagonal 


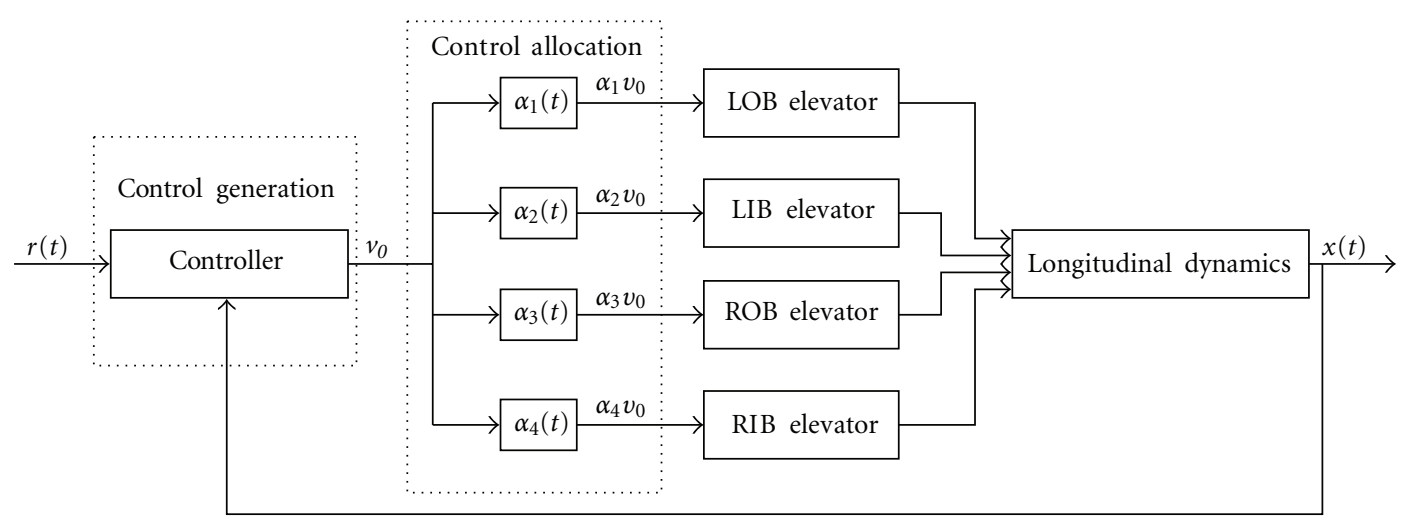

FIgURE 1: Aircraft pitch control with adaptive control allocation.

control effectiveness matrix with $\Lambda=\operatorname{diag}\left\{\lambda_{1}, \lambda_{2}, \ldots, \lambda_{m}\right\}$, and $0<\lambda_{i} \leq 1, i=1, \ldots, m$. The $i$ th actuator is fully functional when $\lambda_{i}=1$ and has a loss of effectiveness failure when $0<\lambda_{i}<1$. For the design in this section, we assume that $\lambda_{i} \neq 0$, that is, no actuator outage occurs. For the reminder of this section we assume that $\Lambda$ is always positive definite. by

The control input $v(t)=\left[v_{1}(t), v_{2}(t), \ldots, v_{m}(t)\right]^{\top}$ is given

$$
v_{j}(t)=\alpha_{j}(t) v_{0}(t), \quad j=1,2, \ldots, m,
$$

where $v_{0}(t)$ is a control signal designed for the group, and $\alpha_{j}(t)$ is the adaptive allocation gain for $j$ th actuator. We assume that a nominal allocation scheme has been prescribed. This scheme will be enforced under nominal operating conditions. The nominal allocation gain vector is denoted as $\alpha^{*} \in R^{m}$. We also define the equivalent control gain vector as $b_{0} \triangleq B \alpha^{*}$. The vector $b_{0}$ can be seen as the equivalent control gain matrix for the equivalent control effector representing the actuator group. We further assume that the pair $\left(A, b_{0}\right)$ is stabilizable.

2.1.2. Reference Model. For the adaptive control, the desired closed-loop dynamics is given by

$$
\dot{x}_{m}(t)=A_{m} x_{m}(t)+B_{m} r(t),
$$

where $A_{m} \in R^{n \times n}$ is a Hurwitz matrix, and $B_{m} \in R^{n}$. The signal $r(t) \in R$ is a bounded piecewise continuous reference input, and $x_{m}(t)$ is the desired state. For a given symmetric positive definite matrix $Q \in R^{n \times n}$, there exists a unique $P \in$ $R^{n \times n}$ that satisfies

$$
P A_{m}+A_{m}^{\top} P=-Q, \quad P=P^{\top}>0 .
$$

For the adaptive control design, we need the following standard plant model matching condition.

Assumption 1. There exist constant $K_{1}^{*} \in R^{n}, K_{2}^{*} \in R$ such that

$$
A+b_{0} K_{1}^{* \top}=A_{m}, \quad b_{0} K_{2}^{*}=B_{m} .
$$

2.1.3. Control Objective. The control objective is to design the virtual control signal $v_{0}(t)$ and adaptive allocation gains $\alpha_{j}, j=1, \ldots, m$, such that all the closed-loop signals are bounded and the system state $x(t)$ tracks the desired state $x_{m}(t)$ asymptotically in the presence of uncertain control effectiveness $\Lambda$.

\subsection{Adaptive Control Allocation Design}

2.2.1. Nominal Controller. The plant model matching condition in Assumption 1 indicates the existence of a nominal controller $v^{*}(t)$ for the system without failures and a nominal constant allocation gain vector $\alpha^{*}$ such that the closedloop response is identical to that of the reference model when the responses of any unmatched initial conditions vanish exponentially. This signal takes the form

$$
\begin{gathered}
v_{0}^{*}(t)=K_{1}^{* \top} x(t)+K_{2}^{*} r(t) \triangleq \theta^{* \top} \omega(t), \\
v^{*}(t)=\alpha^{*} v_{0}^{*}(t),
\end{gathered}
$$

where $\theta^{*} \triangleq\left[K_{1}^{* \top}, K_{2}^{*}\right]^{\top} \in R^{n+1}$, and $\omega(t)=\left[x^{\top}(t), r(t)\right]^{\top}$. The above state feedback control $v_{0}^{*}(t)$ together with a prespecified distribution $\alpha^{*}$ ensures the state tracking error $e(t)=x(t)-x_{m}(t)$ approaches zero exponentially.

2.2.2. Adaptive Controller. When a failure occurs, the allocation gain will be adaptively adjusted to accommodate for failure, but $\alpha^{*}$ may no longer be effective. For this, we use the adaptive versions of control signal and allocation gain

$$
\begin{gathered}
v_{0}(t)=K_{1}^{\top}(t) x(t)+K_{2}(t) r(t)=\theta^{\top}(t) \omega(t), \\
v(t)=\alpha(t) v_{0}(t),
\end{gathered}
$$

where $K_{1}(t)$ and $K_{2}(t)$ are estimates of $K_{1}^{*}$ and $K_{2}^{*}$, and $\theta(t)=\left[K_{1}^{\top}(t), K_{2}(t)\right]^{\top} \in R^{n+1}$. The updated $\alpha(t)=$ $\left[\alpha_{1}(t), \ldots, \alpha_{m}(t)\right]^{\top}$ is an estimate of $\alpha^{*}$. 
2.2.3. Error Dynamics. With the plant and control in (1) and (2), nominal controller in (7), and adaptive controller in (8), the closed-loop dynamics can be expressed as

$$
\begin{aligned}
\dot{x}(t)= & A x(t)+B \Lambda \alpha(t) v_{0}(t) \\
= & A x(t)+B \alpha^{*} v_{0}(t)+B \tilde{\alpha}(t) v_{0}(t) \\
= & A x(t)+b_{0} v_{0}(t)+B \tilde{\alpha}(t) v_{0}(t) \\
= & A x(t)+b_{0} v_{0}^{*}(t)+b_{0} \tilde{\theta}^{\top}(t) \omega(t)+B \tilde{\alpha}(t) v_{0}(t) \\
= & \left(A+b_{0} K_{1}^{* \top}\right) x(t)+b_{0} K_{2}^{*} r(t)+b_{0} \tilde{\theta}^{\top}(t) \omega(t) \\
& +B \tilde{\alpha}(t) v_{0}(t) \\
= & A_{m} x(t)+B_{m} r(t)+b_{0} \tilde{\theta}^{\top}(t) \omega(t)+B \tilde{\alpha}(t) v_{0}(t),
\end{aligned}
$$

where $\tilde{\alpha}(t) \triangleq \Lambda \alpha(t)-\alpha^{*}(t)$ and $\tilde{\theta}(t)=\theta(t)-\theta^{*}$.

From the closed-loop dynamics in (9) and reference model in (4), the error dynamics can be obtained as

$$
\dot{e}(t)=A_{m} e(t)+b_{0} \tilde{\theta}^{\top}(t) \omega(t)+B \tilde{\alpha}(t) v_{0}(t) .
$$

It can be seen that the error dynamics in (10) is suitable for adaptive law design in that the latter half of its right-hand side is linear in $\tilde{\theta}(t)$ and $\tilde{\alpha}(t)$.

2.2.4. Adaptive Laws. Based on the error dynamics in (10), we can design the adaptive laws for the control parameter $\theta(t)$ and allocation gain $\alpha(t)$ as

$$
\begin{gathered}
\dot{\theta}(t)=-\Gamma_{\theta} \omega(t) e^{\top}(t) P b_{0}, \\
\dot{\alpha}(t)=-\Gamma_{\alpha} B^{\top} P e(t) v_{0}(t),
\end{gathered}
$$

where $\Gamma_{\theta}$ and $\Gamma_{\alpha}$ are symmetric positive definite matrices and $P$ is determined by (5). From the adaptive laws, we can see that the controller parameters of the virtual control signal for the actuator group are updated using the information of the equivalent control gain vector $b_{0}$. The allocation gains are updated with the $B$ matrix since successful allocation of the virtual control signal to each actuator requires the knowledge of each column of $B$.

The properties of the adaptive control allocation scheme can be summarized in the following theorem whose proof is presented in the appendix.

Theorem 1. For the system in (1), the adaptive controller and allocation scheme in (8), and the adaptive laws in (11) and (12) guarantee that the all the closed-loop signals are bounded and $\lim _{t \rightarrow \infty}\left[x(t)-x_{m}(t)\right]=0$ in the presence of uncertain loss of effectiveness actuator failures in (2).

Remark 2. From the definition of $\tilde{\alpha}(t)$ in (9), we can see that the adaptation of $\alpha(t)$ is essential for compensating the uncertainties in $\Lambda$, while $K_{1}(t)$ and $K_{2}(t)$ can be fixed to their nominal values $K_{1}^{*}$ and $K_{2}^{*}$. In this case, with $\tilde{\theta}(t)=0$ in (10) and (A.1), the closed-loop stability and asymptotic state tracking results still hold.

\subsection{Examples}

2.3.1. Linear Plants. Two case studies based on linearized aircraft models are presented next.

Plant. Consider the linearized lateral dynamic model of a large transport aircraft flying in a steady wings-level cruise condition with $u=778 \mathrm{ft} / \mathrm{s}$ [15]. The aircraft model is

$$
\begin{gathered}
\dot{x}=A x+B u \\
x=\left[v_{b}, p_{b}, r_{b}, \phi, \psi\right]^{\top}, \quad u=\left[\delta_{a}, \delta_{r}\right]^{\top} .
\end{gathered}
$$

The state includes the lateral velocity $v_{b}(\mathrm{ft} / \mathrm{s})$, roll rate $p_{b}$ $(\mathrm{rad} / \mathrm{s})$, yaw rate $r_{b}(\mathrm{rad} / \mathrm{s})$ (all in body-axis frame), roll angle $\phi(\mathrm{rad})$, and yaw angle $\psi(\mathrm{rad})$. The control inputs are aileron $\delta_{a}$ and rudder $\delta_{r}$ deflections (deg). The system matrices $A$ and $B$ are

$$
\begin{gathered}
A=\left[\begin{array}{ccccc}
-0.129 & 28.328 & -774.92 & 32.145 & 0 \\
-0.012 & -1.4419 & 0.9409 & 0 & 0 \\
0.004 & -0.0409 & -0.1757 & -0.0001 & 0 \\
0 & 1 & 0.0372 & 0 & 0 \\
0 & 0 & 1.0007 & 0 & 0
\end{array}\right], \\
B=\left[\begin{array}{cc}
0.0542 & 0.4669 \\
0.0443 & 0.0200 \\
0.0025 & -0.0382 \\
0 & 0 \\
0 & 0
\end{array}\right] .
\end{gathered}
$$

Hereafter we will group the aileron and rudder into a single group. Note that these surfaces have related but different functionalities. If adaptive control signals are designed separately for the two control inputs with the state feedback structure, the total updated parameters would be $(5+1) \times 2=$ 12. They include 5 state feedback parameters and 1 feedforward parameter (for tracking the reference input) for each control surface. The adaptive control allocation scheme, on the other hand, requires $5+1+2=8$ parameters since only one adaptive control signal is designed and allocated with two updated allocation gains.

Nominal Parameters. The nominal allocation gain vector is selected as $\alpha^{*}=[8.6103,-1]^{\top}$, thus the equivalent control gain vector is $b_{0}=B \alpha^{*}=[0,0.3614,0.0594,0,0]^{\top}$. The nominal allocation gain is chosen so that the first element in $b_{0}$, that is, the control gain for the lateral acceleration is zero. The purpose of this choice is to attain a coordinated turn.

The nominal controller is designed based on the LQR approach for $\left(A, b_{0}\right)$. The resulting gains are

$$
\begin{gathered}
K_{1}^{*}=[-0.8963,22.1655,-73.6645,28.8488,4.0825]^{\top}, \\
K_{2}^{*}=1 .
\end{gathered}
$$

Reference Model. For this example, the reference model is chosen as the closed-loop dynamics with the above LQR controller, that is,

$$
A_{m}=A+b_{0} K_{1}^{* \top}, \quad B_{m}=b_{0} K_{2}^{*} .
$$


The reference input to the reference model is chosen as $r(t)=$ 2.1376 for $t \geq 0$, which leads to a desired state trajectory with steady-state values

$$
x_{m}(\infty)=[0,0,0,0,0.5236]^{\top} .
$$

This reference command yields a right turn with a change in the yaw angle of 30 degrees.

Actuator Failure. We will consider the $80 \%$ loss in control effectiveness:

$$
u_{1}(t)=0.2 v_{1}(t), \quad \text { for } t \geq 10 \text { seconds, }
$$

where $u_{1}(t)$ is the output of the aileron actuator and $v_{1}(t)$ is the designed control input to aileron.

Simulation Results. The following cases will be studied.

(i) Case 1: adaptive allocation of the adaptive control designed in Section 2.2 ((11) and (12)).

(ii) Case 2: adaptive allocation (as in (12)) of a nonadaptive control signal with fixed gains (as in (16)).

Case 1. The time history of the system state under failure and the desired state is shown in Figure 2. It can be seen that the asymptotic state tracking is achieved after failure.

Figure 3 shows the designed control signal $v_{0}(t)$, allocated control signals $v(t)$ and actuator outputs $u(t)$. The designed control signal is allocated by the adaptive allocation gains. The actuator outputs are different from the allocated control signals due to the failure.

The adaptive parameters are shown in Figure 4. We can see that $K_{2}(t)$ also plays an important role in the compensation of actuator failures. The adaptive allocation gains are shown in Figure 5. Both allocations gains adapt immediately after the failure occurs and settle to a new combination of steady-state values that, together with $K_{2}(t)$ and $K_{1}(t)$, guarantee state tracking after failure.

Case 2. The system state and desired state are shown in Figure 6. Despite the uncertain actuator failure, asymptotic state tracking is achieved. The designed control signal, allocated control signals and the actuator outputs are shown in Figure 7. Similar to the previous case, the allocated control signals and the actuator outputs are not identical due to the actuator failure. The allocation gains are shown in Figure 8. The allocation gains are updated autonomously for failure compensation.

In this simulation case, we have achieved similar results to Case 1 with a slightly degraded transient response (see Figures 2 and 6). A possible explanation for it is that the controller parameters are fixed in this case and cannot contribute to the failure compensation and trajectory tracking as they do in Case 1. However, closed-loop stability and asymptotic state tracking are achieved even though only the allocation gains are adapted. The successful demonstration of failure compensation in Case 2 implies that the proposed adaptive allocation unit can be added to a control loop having any
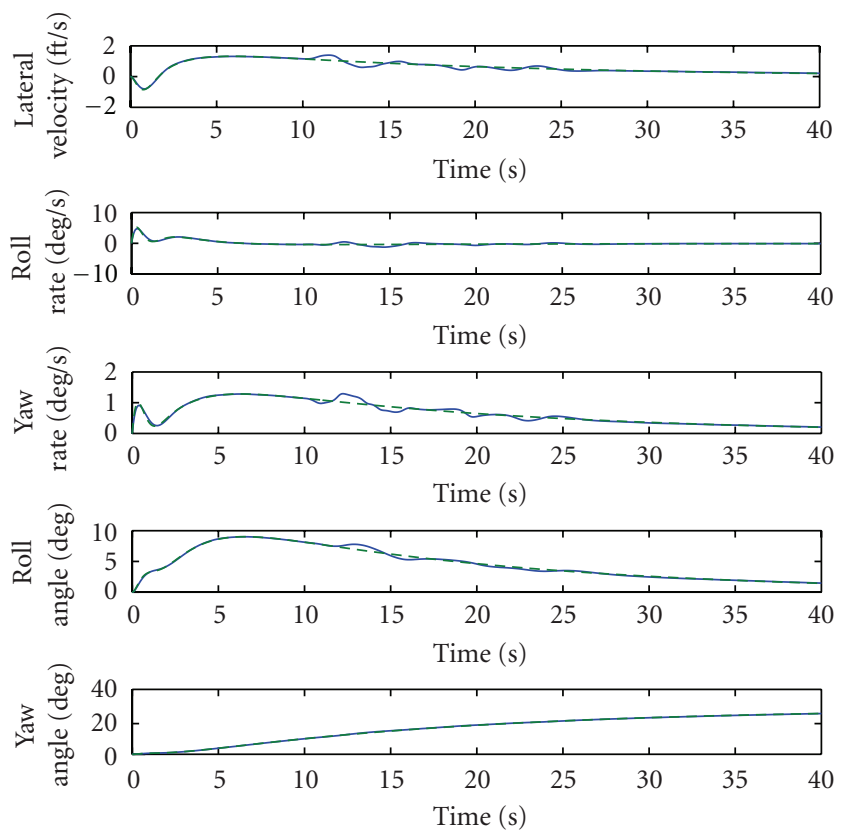

FIGURE 2: Time history of plant state (solid) and reference model state (dashed) (Case 1).
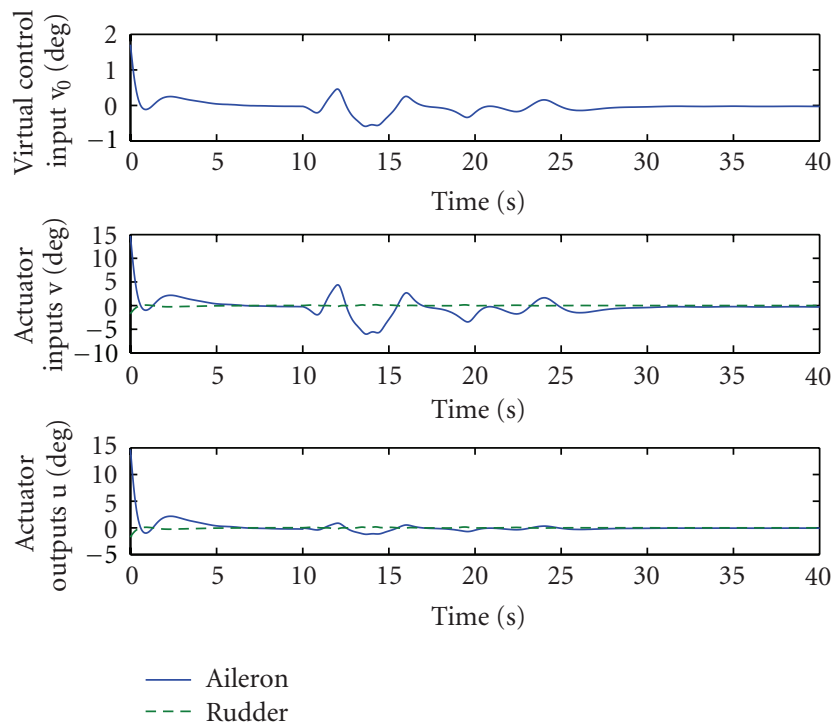

Figure 3: Time history of control signals and actuator outputs (Case 1).

state feedback controller. The added adaptive allocation to the nonadaptive controller ensures closed-loop stability and asymptotic state tracking despite uncertain actuator failures.

2.3.2. NASA Generic Transport Model. In this section, we apply the control allocation strategy to the NASA Generic Transport Model (GTM). The NASA GTM is a high-fidelity model of the NASA AirSTAR UAV testbed $[16,17]$. The purpose of this example is to show that this adaptive scheme, with the grouping of actuators having different physical 

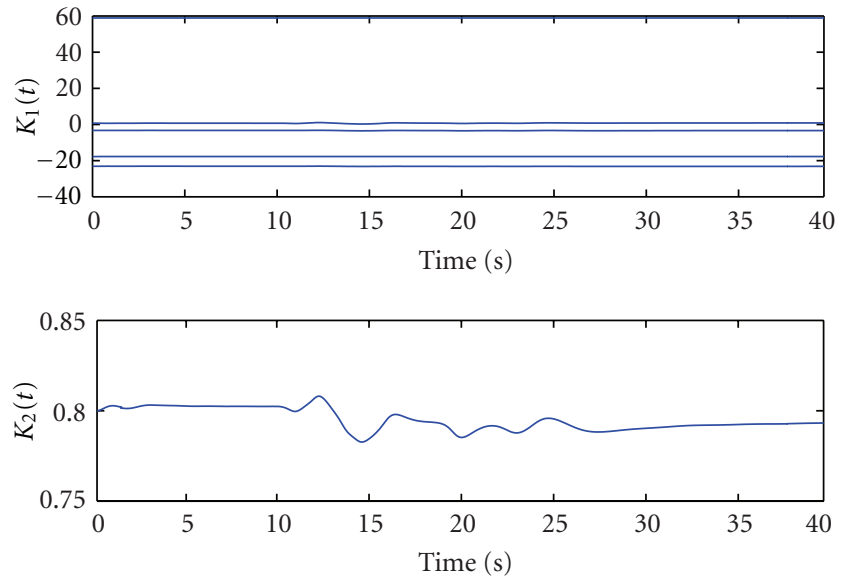

FIgURE 4: Time history of controller parameters (Case 1).

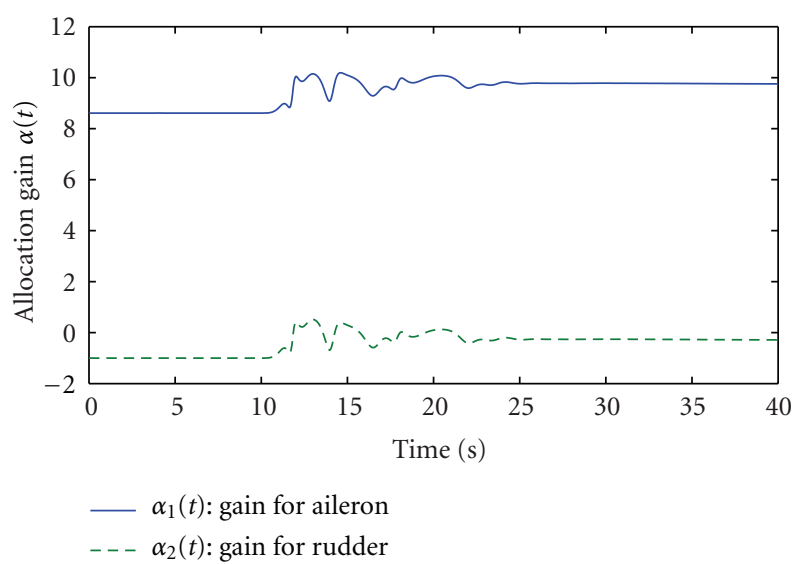

Figure 5: Time history of allocation gains (Case 1).
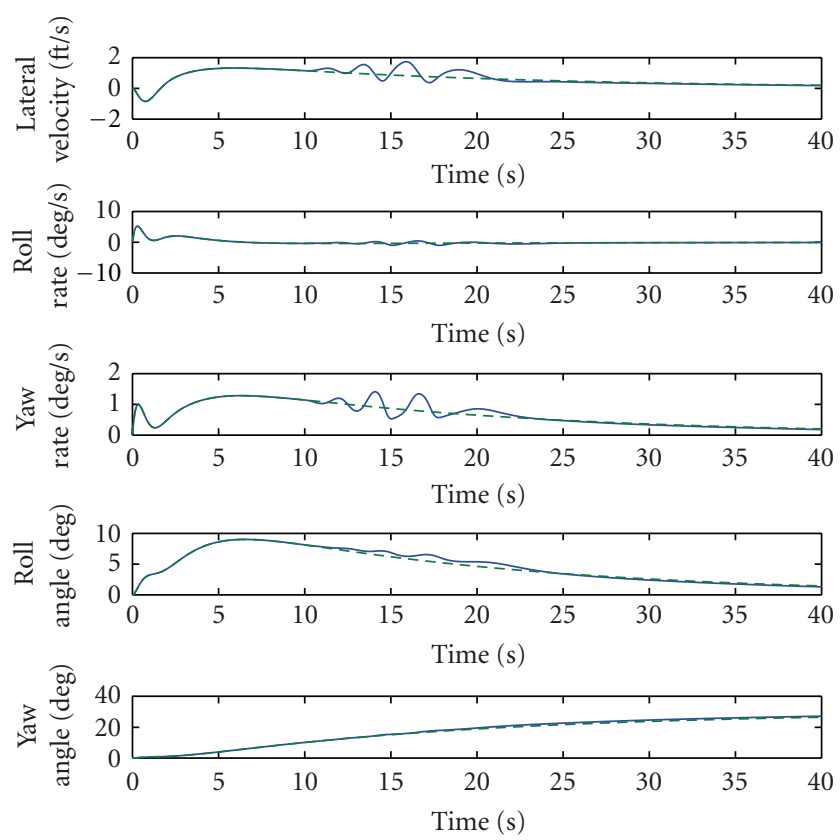

Figure 6: Time history of plant state (solid) and reference model state (dashed) (Case 2).
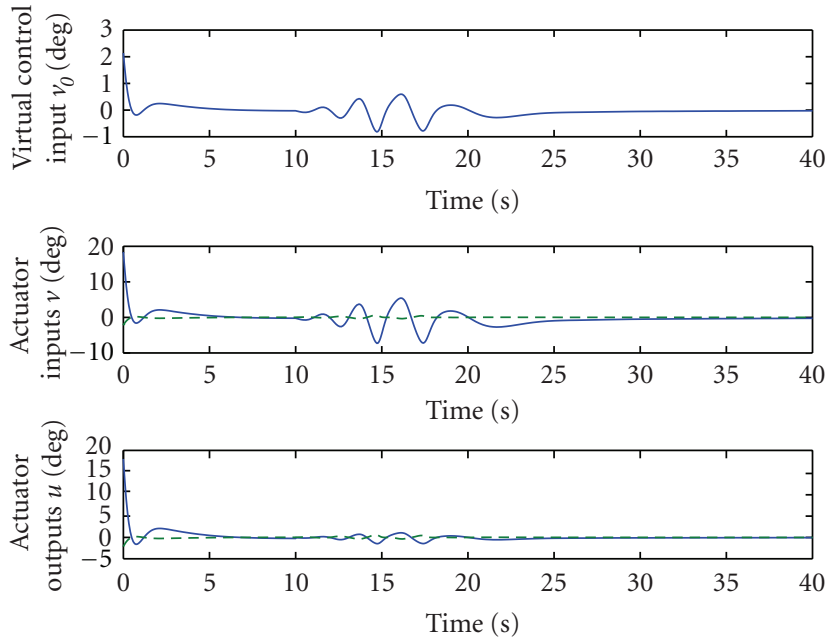

- Aileron
-- Rudder

Figure 7: Time history of control signals and actuator outputs (Case 2).

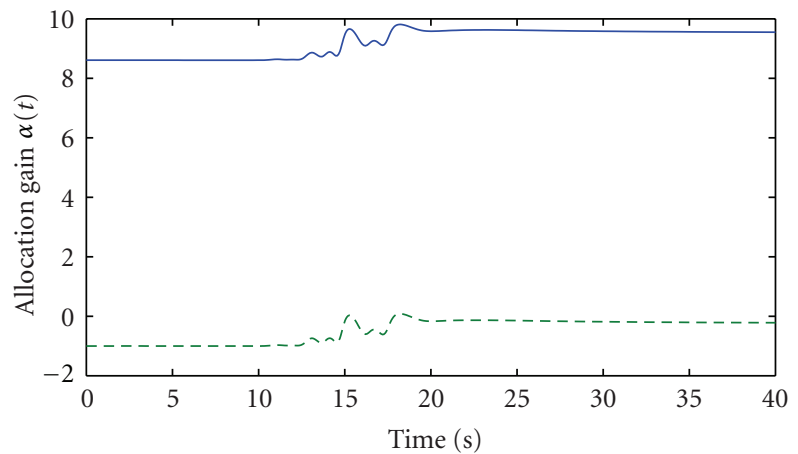

$\alpha_{1}(t)$ : gain for aileron

- - $\alpha_{2}(t)$ : gain for rudder

FIGURE 8: Time history of allocation gains (Case 2).

functions, can be applied to the nonlinear plant to achieve closed-loop stability and asymptotic tracking.

LTI Model. For this simulation study, we trim and linearize the GTM at a wings-level flight for an aerodynamic speed of 92.09 knots. The same states and controls of (14) are used.

Flight Conditions. As in the previous example, the aircraft is commanded to turn right from the initial wings-level horizontal flight. The turn starts at 10 seconds and at steady state the heading angle will increase 60 degrees.

Actuator Failure. We let the left aileron lose $90 \%$ of its effectiveness at 12 seconds. The failure magnitude and its onset time instant are unknown to the controller. The control objective is for the aircraft to achieve an accurate turn in the presence of the failure. 

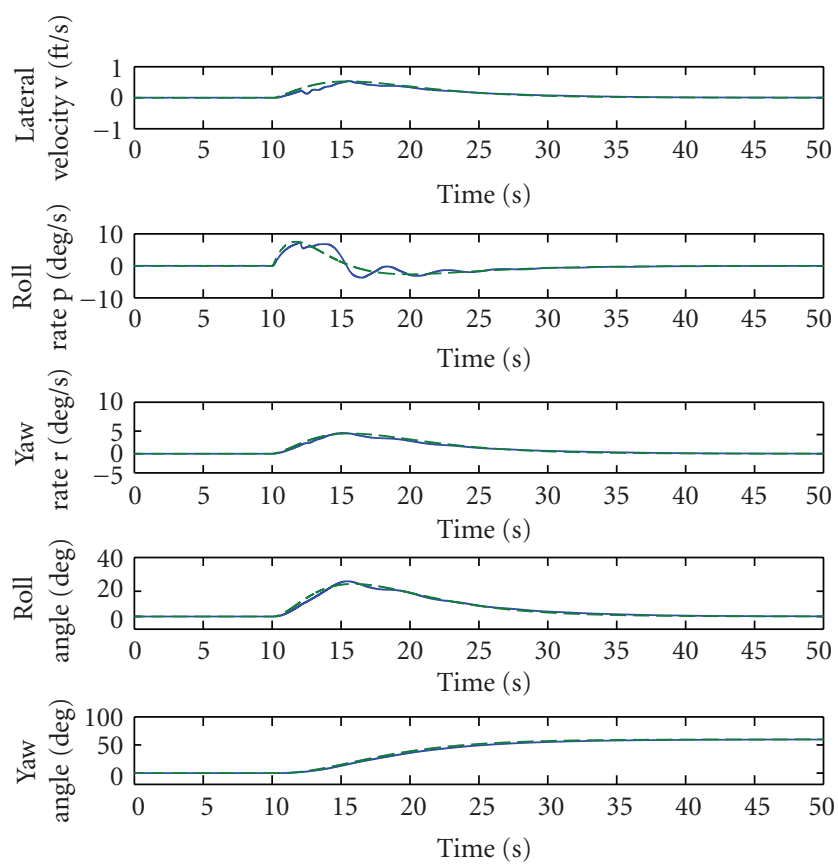

FIGURE 9: Time history of lateral states (solid) and reference model states (dashed).
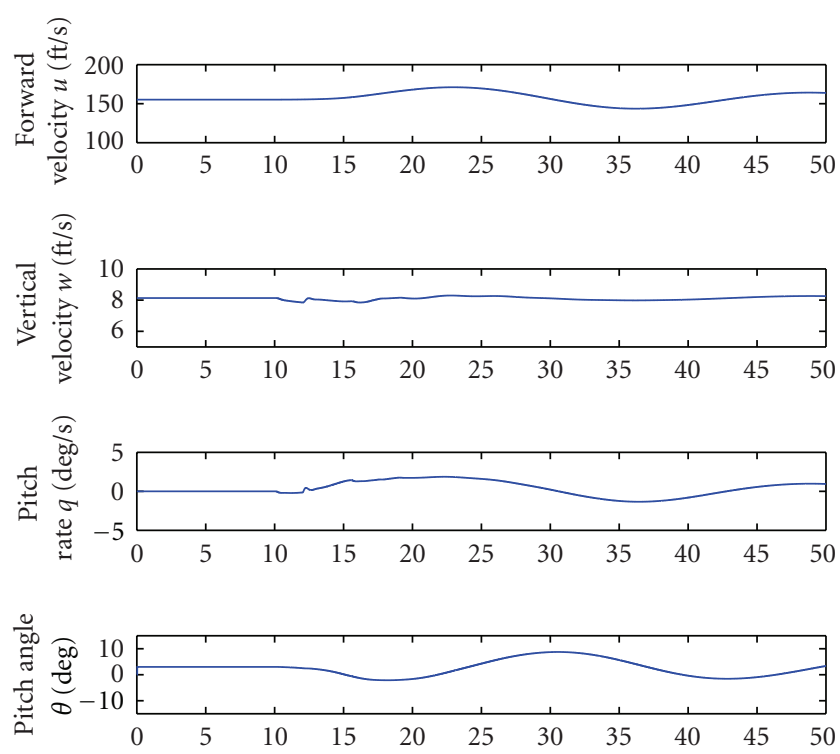

FIGURE 10: Time history of longitudinal states.

Simulation Results. The simulation results are shown in Figures 9-14. Figure 9 shows the relevant states of the reference model and of the plant. The states track the reference trajectories asymptotically in spite of the disturbance caused by the failure. The yaw angle is shown to reach 60 degrees accurately. This accurate turning is also shown in Figure 11 by the ground track of the aircraft.

The longitudinal states are shown in Figure 10. There are fluctuations in the longitudinal states since they are not controlled by aileron and rudder.

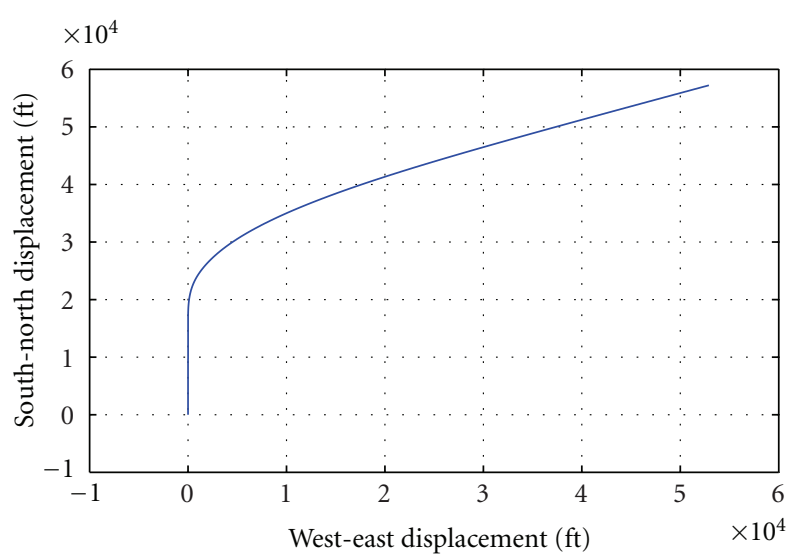

FIGURE 11: Ground track of the aircraft.
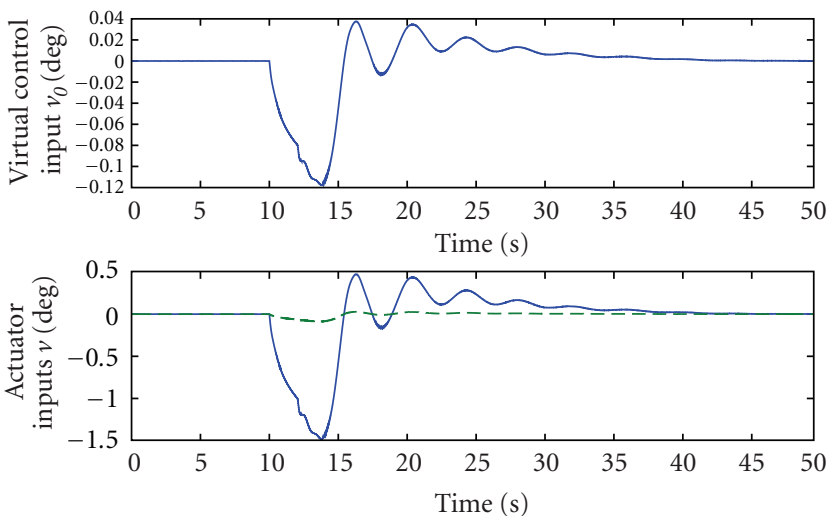

- Aileron
-- - Rudder

FIgURE 12: Time history of virtual control signal and actuator inputs.

Figures 12 and 13 show the time history of the virtual control signal, allocated control signals, and actual aileron and rudder deflections. The discontinuity in Figure 13 at the top is a consequence of failure.

Figure 14 shows the control allocation gains and controller parameters. The parameters adjust autonomously after the failure occurs. Note that the allocation gains start to update at the beginning of the turn at $t=10$ seconds, before the failure occurs. The reason for this phenomenon is that the allocation gains are improving the tracking performance beyond of what the adaptive gains can do alone.

\section{Adaptive Control Allocation Design for Constant Failures}

3.1. Problem Formulation. When constant failures occur, the control signal can be rewritten as [11]

$$
u(t)=v(t)+\sigma_{f}(\bar{u}-v(t))
$$




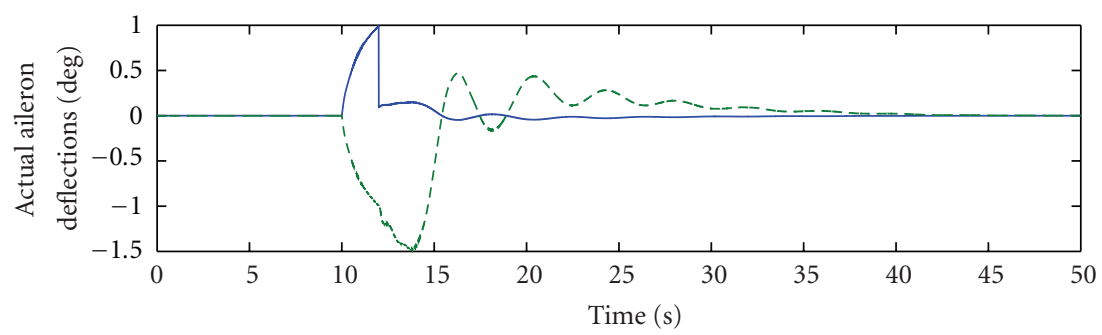

— Left aileron

- - - Right aileron

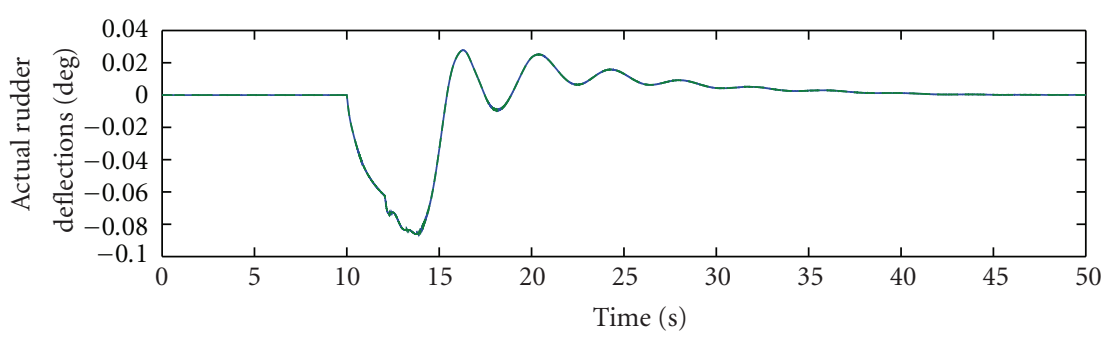

- Upper rudder

- - - Lower rudder

FIgURE 13: Time history of actuator deflections.
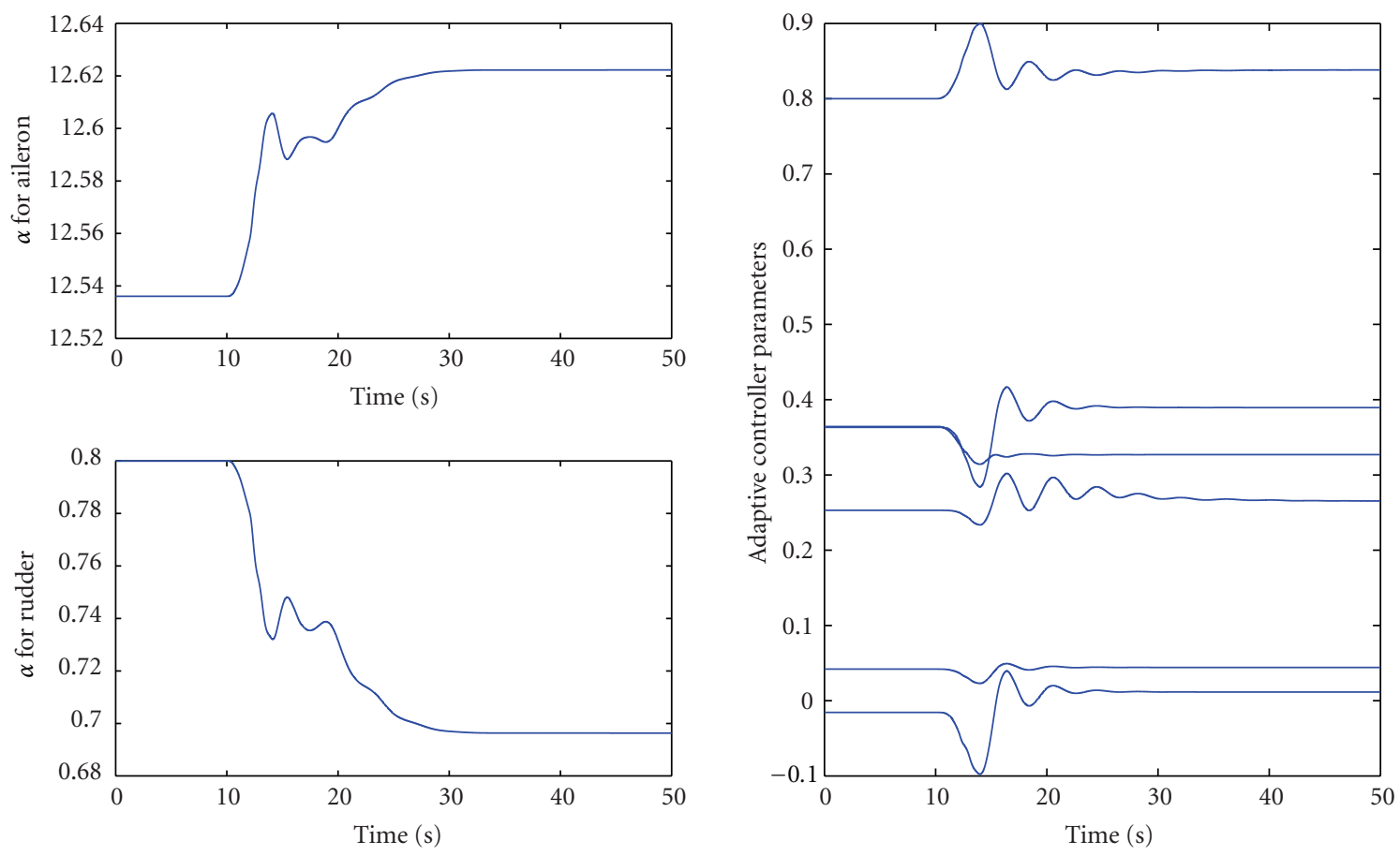

FIGURE 14: Time history of allocation gains and adaptive parameters.

where $\bar{u}=\left[\bar{u}_{1}, \ldots, \bar{u}_{m}\right]^{\top}$ is the failure vector whose elements are unknown constants, and $\sigma_{f}$ represents the failure pattern, which is defined as

$$
\sigma_{f}=\operatorname{diag}\left\{\sigma_{1}, \sigma_{2}, \ldots, \sigma_{m}\right\},
$$

with $\sigma_{i}=1$ if the $i$ th actuator has failed, that is, $u_{i}=\bar{u}_{i}$, and $\sigma_{i}=0$ otherwise. The failures are assumed to occur instantaneously, that is, $\sigma_{i}$ are piecewise constant function of time. An example of such actuator failures is when a control surface (such as the rudder or an aileron) is stuck at some unknown fixed angle at an unknown time instant. This type 
of failures could be caused by failed hydraulic systems or mechanical linkages.

The plant dynamics can then be rewritten as

$$
\dot{x}(t)=A x(t)+B\left(I-\sigma_{f}\right) v(t)+B \sigma_{f} \bar{u} .
$$

The constant failure $\bar{u}$ introduces an uncertain disturbance that needs to be accommodated for. The control objective for this adaptive control allocation scheme is to design $v(t)$ to guarantee closed-loop stability and asymptotic state tracking when uncertain constant failures occur.

For adaptive control of constant actuator failures, sufficient built-in actuation redundancy is required. The redundancy condition is described in the following assumption.

Assumption 2. The rank of $B$ matrix satisfies that $\operatorname{rank}[B]=$ 1 , and there is at least one operable actuator in the system.

The rank condition characterizes the redundancy of actuation which is necessary for a successful constant failure compensation. This rank condition suggests that the system remains controllable after a failure, and the effect of the constant failure can be properly matched and canceled by the allocated control signals through other columns of $B$. Based on this condition, there can be up to $m-1$ constant actuator failures at any given time.

3.1.1. Nominal Controller. Consider the nominal controller structure

$$
\begin{gathered}
v_{0}^{*}(t)=K_{1}^{* \top} x(t)+K_{2}^{*} r(t)+K_{3}^{*} \triangleq \bar{\theta}^{* \top} \bar{\omega}(t), \\
v^{*}(t)=\alpha^{*} v_{0}^{*}(t),
\end{gathered}
$$

where $\bar{\theta}^{*}=\left[K_{1}^{* \top}, K_{2}^{*}, K_{3}^{*}\right]^{\top}$ and $\bar{\omega}=\left[x^{\top}(t), r(t), 1\right]^{\top}$. When there is no failure in the system, $K_{1}^{*}, K_{2}^{*}$, and $\alpha^{*}$ are chosen as in (6), and $K_{3}^{*}$ is set to be zero. In this way, the controller ensures the match between the reference model and the nominal plant without failures. Similar to the previous section, we also define $B \alpha^{*}=b_{0}$ which is known in advance for controller design.

Next we will show that the controller in (23) attains model matching when failures occur. When there are failures in the system, $K_{3}^{*}$ cannot generally be zero, and a new set of allocation gains, denoted as $\bar{\alpha}^{*}$, may be needed. From (22) and (23) we obtain

$$
\dot{x}=A x+B\left(I-\sigma_{f}\right) \bar{\alpha}^{*}\left(K_{1}^{* \top} x+K_{2}^{*} r+K_{3}^{*}\right)+B \sigma_{f} \bar{u} .
$$

We assume that at most $p$ actuators can fail with $p \leq m-1$ so that there is at least one operable actuator left. Define an index set for failed actuators as $\mathcal{F}=\left\{i_{1}, \ldots, i_{p}\right\}$ such that $\sigma_{k}=1$ for any $k \in \mathcal{F}$. Then $B\left(I-\sigma_{f}\right) \bar{\alpha}^{*}$ can be expressed as

$$
B\left(I-\sigma_{f}\right) \bar{\alpha}^{*}=\sum_{j \notin \mathcal{F}} b_{j} \bar{\alpha}_{j}^{*},
$$

where $b_{j}$ is the $j$ th column of $B$ and $\bar{\alpha}_{j}^{*}$ is the $j$ th element of $\bar{\alpha}^{*}$.

Based on the rank condition in Assumption 2, we know that the columns of $B$ are linearly dependent, that is, for any two columns of $B, b_{i}$ and $b_{j}, b_{i}=c_{i j} b_{j}$ where $c_{i j}$ is a constant scalar. Thus we know that the vector $b_{0}=B \alpha^{*}$, which is the linear combination of all columns of $B$, is also parallel to any column in $B$. So for each column $b_{k}$ in $B, k=1,2, \ldots, m$, we can find a scalar $c_{k}$ satisfying $b_{k}=c_{k} b_{0}$. Therefore (25) can be expressed as

$$
B\left(I-\sigma_{f}\right) \bar{\alpha}^{*}=\sum_{j \notin \mathcal{F}} b_{j} \bar{\alpha}_{j}^{*}=\sum_{j \notin \mathcal{F}} c_{j} \bar{\alpha}_{j}^{*} b_{0} .
$$

If $\bar{\alpha}_{j}^{*}, j \notin \mathcal{F}$ are chosen such that

$$
\sum_{j \notin \mathcal{F}} c_{j} \bar{\alpha}_{j}^{*}=1,
$$

then we may obtain

$$
B\left(I-\sigma_{f}\right) \bar{\alpha}^{*}=b_{0} .
$$

One possible choice for $\bar{\alpha}_{j}^{*}, j \notin \mathcal{F}$ is

$$
\bar{\alpha}_{j}^{*}=\frac{1}{c_{j}(m-p)} .
$$

Equation (28) indicates that, under constant failures, the plant model condition in (6) can still be satisfied. Note that for the system without failures

$$
B \alpha^{*}=\sum_{j=1}^{m} \alpha_{j}^{*} b_{j}=\sum_{j=1}^{m} c_{j} \alpha_{j}^{*} b_{0}=b_{0}
$$

which implies that

$$
\sum_{j=1}^{m} \mathrm{c}_{j} \alpha_{j}^{*}=1
$$

Comparing (27) and (31), we can see that $\bar{\alpha}^{*}$ is generally different from $\alpha^{*}$.

For $B \sigma_{f} \bar{u}$, we can also get

$$
B \sigma_{f} \bar{u}=\sum_{k \in \mathcal{F}} b_{k} \bar{u}_{k}=\sum_{k \in \mathcal{F}} c_{k} \bar{u}_{k} b_{0} \triangleq d^{*} b_{0} .
$$

With (28) and (32), (24) can be expressed as

$$
\dot{x}(t)=A x(t)+b_{0} K_{1}^{* \top} x(t)+b_{0} K_{2}^{*} r(t)+b_{0} K_{3}^{*}+d^{*} b_{0} .
$$

By choosing $K_{3}^{*}=-d^{*}$, we have $b_{0} K_{3}^{*}=-B \sigma_{f} \bar{u}$, and (33) can be reduced to

$$
\begin{aligned}
\dot{x}(t) & =A x(t)+b_{0} K_{1}^{* \top} x(t)+b_{0} K_{2}^{*} r(t) \\
& =\left(A+b_{0} K_{1}^{* \top}\right) x(t)+b_{0} K_{2}^{*} r(t) .
\end{aligned}
$$

Therefore when failures are present, a nominal controller can always be found, with $K_{1}^{*}$ and $K_{2}^{*}$ specified in (6), $\bar{\alpha}^{*}$ characterized in (27), and $K_{3}^{*}=-d^{*}$ ensures that the closedloop system is stable under constant failures, and the state converges to the desired state $x_{m}$ in (4) exponentially. 


\subsection{Adaptive Control Allocation Design}

3.2.1. Adaptive Controller. Due to the uncertain nature of the failures, the controller parameters must be adjusted. Consider the adaptive control allocation scheme

$$
\begin{gathered}
v_{0}(t)=K_{1}^{\top}(t) x(t)+K_{2}(t) r(t)+K_{3}(t) \triangleq \bar{\theta}^{\top}(t) \bar{\omega}(t), \\
v(t)=\alpha(t) v_{0}(t)
\end{gathered}
$$

where $K_{1}(t), K_{2}(t)$, and $K_{3}(t)$ are the estimates of $K_{1}^{*}, K_{2}^{*}$, and $K_{3}^{*}$ in (23). The signal $\bar{\omega}(t)=\left[x^{\top}(t), r(t), 1\right]^{\top}$. With the adaptive controller in (35) and the plant dynamics in (22), the closed-loop system is

$$
\begin{aligned}
\dot{x}(t)= & A x(t)+B\left(I-\sigma_{f}\right) \alpha(t) \\
& \times\left[K_{1}^{\top}(t) x(t)+K_{2}(t) r(t)+K_{3}(t)\right]+B \sigma_{f} \bar{u} .
\end{aligned}
$$

3.2.2. Error Dynamics. For this adaptive control scheme design, we define $\tilde{\alpha}(t) \triangleq \alpha(t)-\bar{\alpha}^{*}$, and (36) becomes

$$
\begin{aligned}
\dot{x}(t)= & A x(t)+B\left(I-\sigma_{f}\right) \alpha(t) v_{0}(t)+B \sigma_{f} \bar{u} \\
= & A x(t)+B\left(I-\sigma_{f}\right) \tilde{\alpha}(t) v_{0}(t)+B\left(I-\sigma_{f}\right) \bar{\alpha}^{*} v_{0}(t) \\
& +B \sigma_{f} \bar{u} \\
= & A x(t)+B\left(I-\sigma_{f}\right) \tilde{\alpha}(t) v_{0}(t)+B\left(I-\sigma_{f}\right) \bar{\alpha}^{*} v_{0}^{*}(t) \\
& +B\left(I-\sigma_{f}\right) \bar{\alpha}^{*} \tilde{v}_{0}(t)+B \sigma_{f} \bar{u},
\end{aligned}
$$

where $\widetilde{v}_{0}(t)=\left(\bar{\theta}(t)-\bar{\theta}^{*}\right)^{\top} \bar{\omega}(t) \triangleq \tilde{\theta}^{\top}(t) \bar{\omega}(t)$.

With (28), $B\left(I-\sigma_{f}\right) \bar{\alpha}^{*} v_{0}^{*}(t)$ in (37) becomes

$$
\begin{aligned}
B\left(I-\sigma_{f}\right) \bar{\alpha}^{*} v_{0}^{*}(t)= & b_{0} v_{0}^{*}(t)=b_{0} K_{1}^{* \top} x(t)+b_{0} K_{2}^{*} r(t) \\
& +b_{0} K_{3}^{*} .
\end{aligned}
$$

With (38), (37), and the plant model matching condition in (6), we have

$\dot{x}(t)=A_{m} x(t)+B_{m} r(t)+B\left(I-\sigma_{f}\right) \tilde{\alpha}(t) v_{0}(t)+b_{0} \tilde{\theta}^{\top}(t) \bar{\omega}(t)$.

From the closed-loop dynamics in (39) and the reference model in (4), we obtain the error dynamics

$$
\dot{e}(t)=A_{m} e(t)+B\left(I-\sigma_{f}\right) \tilde{\alpha}(t) v_{0}(t)+b_{0} \tilde{\theta}^{\top}(t) \bar{\omega}(t) .
$$

3.2.3. Adaptive Laws. From the error dynamics in (40), the following adaptive laws are derived:

$$
\begin{gathered}
\dot{\bar{\theta}}(t)=-\Gamma_{\theta} \bar{\omega}(t) e^{\top}(t) P b_{0}, \\
\dot{\alpha}_{j}(t)=-\gamma_{j} e^{\top}(t) P b_{j} v_{0}(t), \quad j=1,2, \ldots, m,
\end{gathered}
$$

where $b_{j}$ is the $j$ th column of $B, \gamma_{j}>0$ and $\Gamma_{\theta}=\Gamma_{\theta}^{\top}>0$ are adaptive gains.

The following theorem summarizes the properties of the adaptive control allocation scheme and the proof is provided in the Appendix:
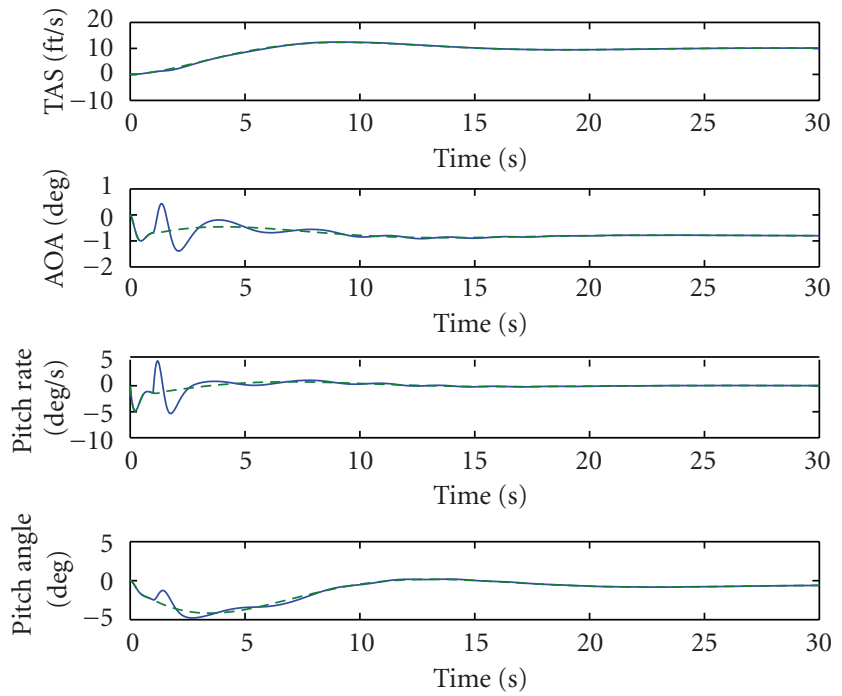

FIGURE 15: Time history of plant state (solid) and reference model state (dashed).
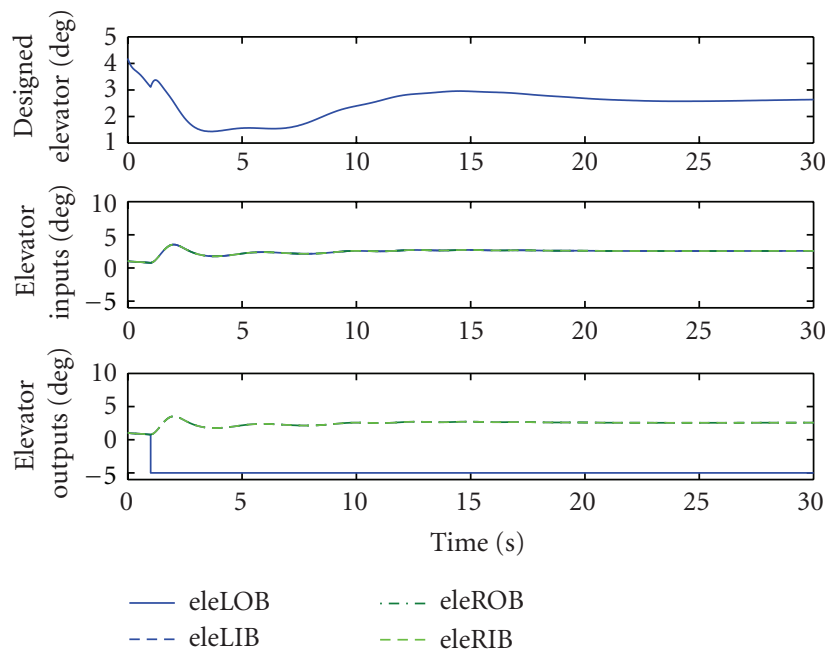

FIgURE 16: Time history of control signals and actuator outputs.

Theorem 3. The adaptive control allocation scheme in (35) with the adaptive laws in (41) and (42) applied to the plant in (22) in the presence of constant failures guarantees that all closed-loop signals are bounded and $\lim _{t \rightarrow \infty}\left(x(t)-x_{m}(t)\right)=0$.

\subsection{Example}

3.3.1. Linear Plant. Consider the longitudinal LTI model of the NASA GTM given by

$$
\begin{gathered}
\dot{x}=A x+B v, \\
x=\left[V_{T}, \alpha_{a}, q, \theta\right]^{\top}, \\
v=\left[\delta_{\text {elob }}, \delta_{\text {elib }}, \delta_{\text {erob }}, \delta_{\text {erib }}\right]^{\top},
\end{gathered}
$$

where the state includes the true airspeed $V_{T}(\mathrm{ft} / \mathrm{s})$, angle of attack $\alpha_{a}(\mathrm{rad})$, pitch rate $q(\mathrm{rad} / \mathrm{s})$, and pitch angle $\theta(\mathrm{rad})$. The control inputs are the deflections of the four elevator 

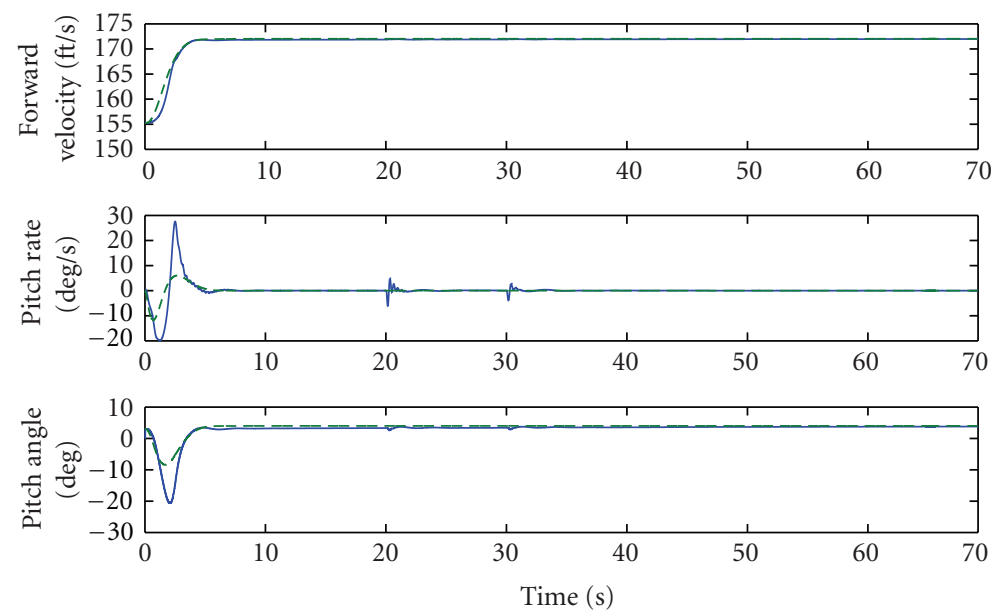

FIGURE 17: Time history of longitudinal states (solid) and reference model states (dashed).
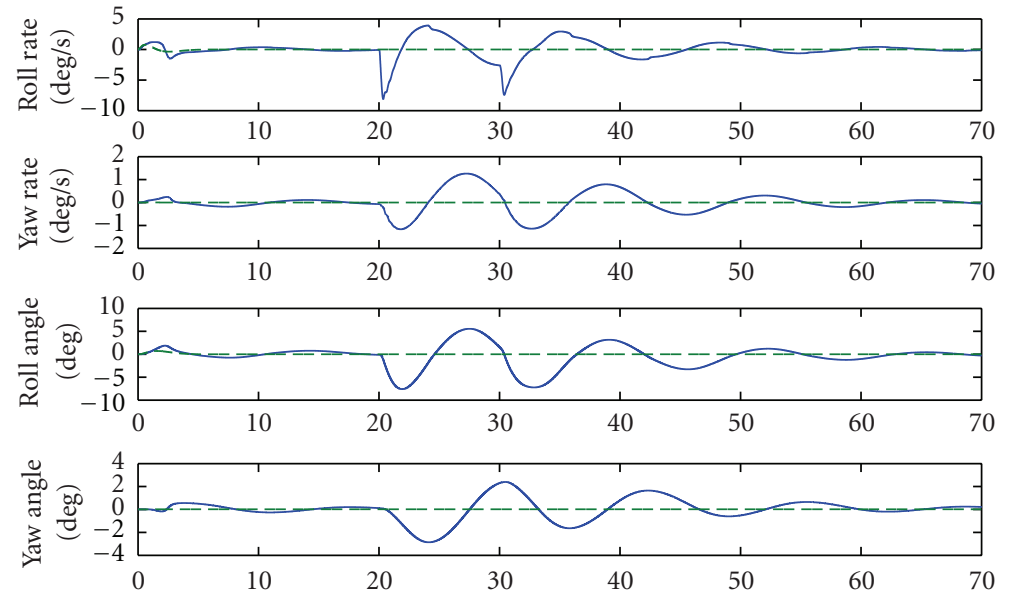

FIGURE 18: Time history of lateral states (solid) and reference model states (dashed).

segments: left outboard elevator $\delta_{\text {elob }}$, left inboard elevator $\delta_{\text {elib }}$, right outboard elevator $\delta_{\text {erob }}$, and right inboard elevator $\delta_{\text {erib }}$ (deg). The system matrices $A$ and $B$ are

$$
\begin{gathered}
A=\left[\begin{array}{cccc}
-0.0450 & -8.9632 & 0.0349 & -32.1740 \\
-0.0035 & -2.7429 & 0.9514 & 0 \\
-0.0056 & -42.6233 & -3.5616 & 0 \\
0 & 0 & 1 & 0
\end{array}\right], \\
B=\left[\begin{array}{cccc}
-0.0110 & -0.0110 & -0.0110 & -0.0110 \\
-0.0012 & -0.0012 & -0.0012 & -0.0012 \\
-0.1962 & -0.1962 & -0.1962 & -0.1962 \\
0 & 0 & 0 & 0
\end{array}\right] .
\end{gathered}
$$

Obviously, $\operatorname{rank}[B]=1$. For the simulation study, we will include the four elevator surfaces into one group, for which an elevator control signal will be designed and allocated.

Nominal Parameters. The nominal allocation gain $\alpha^{*}$ is chosen as $\alpha^{*}=[0.25,0.25,0.25,0.25]^{\top}$ and $b_{0}=B \alpha^{*}=$ $[-0.011,-0.0012,-0.1962,0]^{\top}$ so the deflection of the four segments will be the same if no failure occurs. The nominal controller is designed using the LQR approach for $\left(A, b_{0}\right)$. The resulting gains are

$$
K_{1}^{*}=[0.1494,25.0761,-3.2841,-27.1293]^{\top}, \quad K_{2}^{*}=1 .
$$

Reference Model. Similar to the simulation study in Section 2, the reference model is chosen as the closed-loop dynamics of the LQR controller, that is,

$$
A_{m}=A+b_{0} K_{1}^{* \top}, \quad B_{m}=b_{0} K_{2}^{*} .
$$

The reference input to the reference model is chosen as $r(t)=$ 4.1841 for $t \geq 0$, which leads to a reference trajectory with steady-state values

$$
x_{m}(\infty)=[10,-0.0139,0,-0.0110]^{\top},
$$

whose physical meaning is that the aircraft speed is increased by $10 \mathrm{ft} / \mathrm{s}$; its angle of attack is reduced by $0.0139 \mathrm{rad}$; and its pitch angle is reduced by $0.0110 \mathrm{rad}$. 

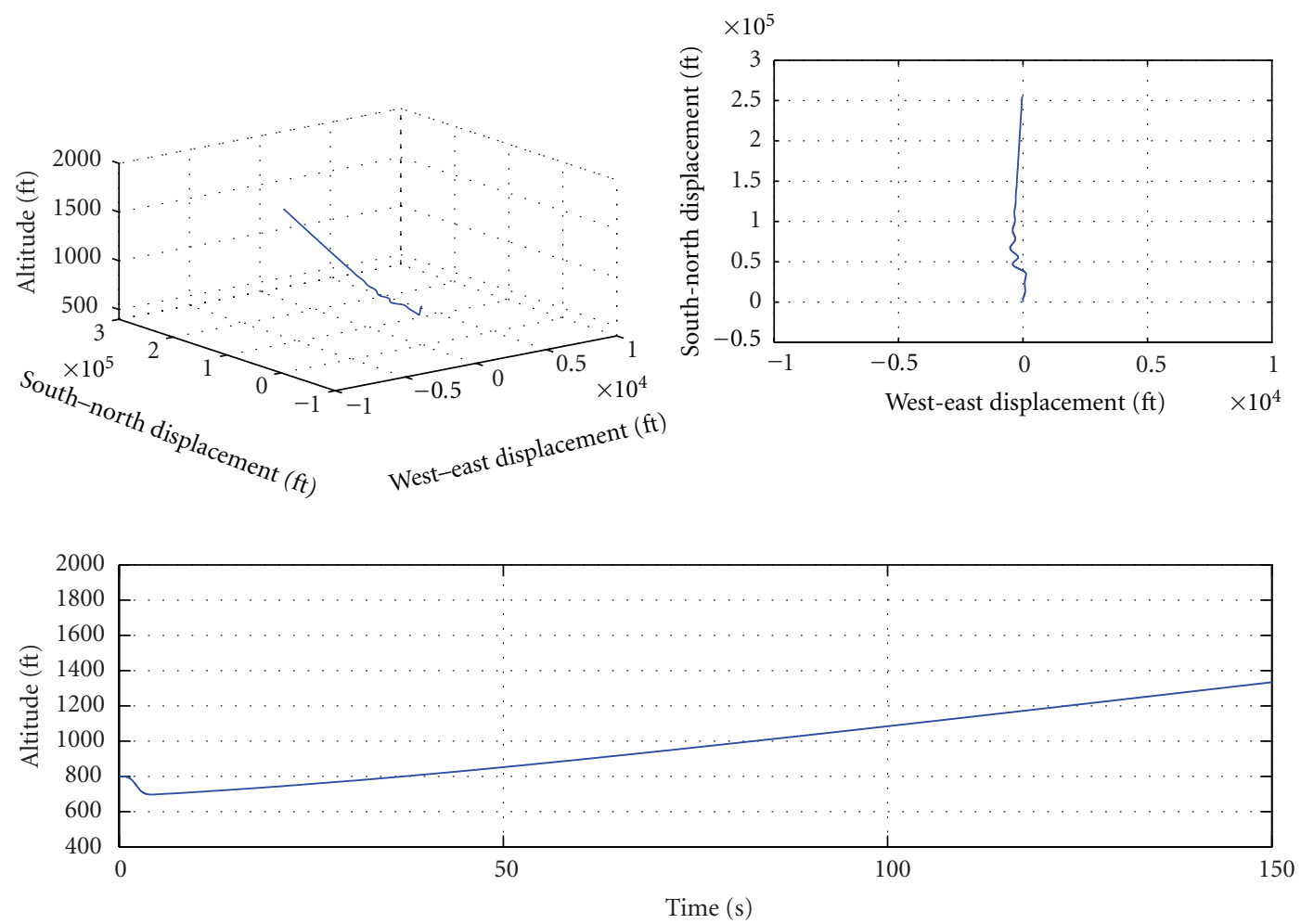

FIGURE 19: Flight trajectory, ground track, and altitude of the aircraft.
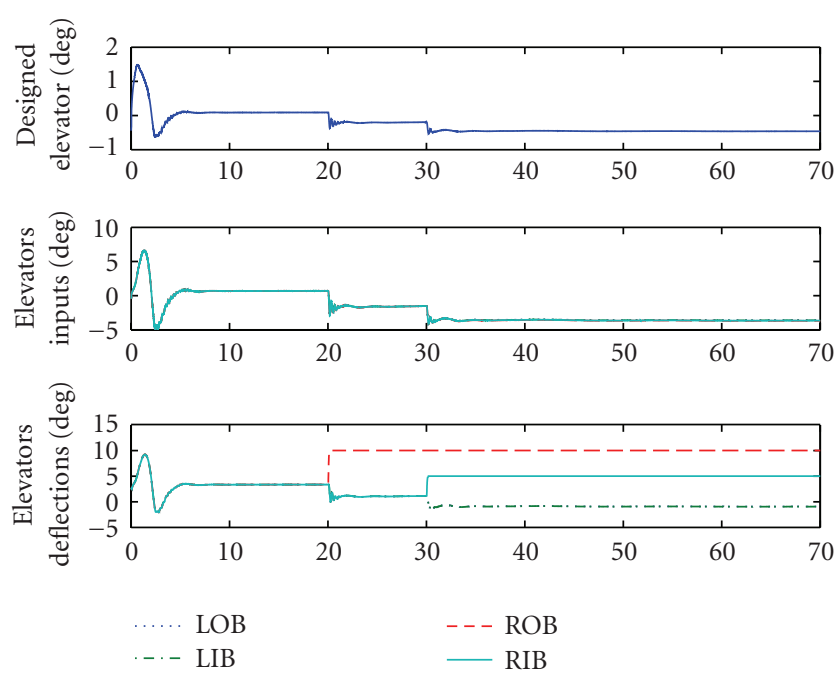

Figure 20: Time history of lumped and allocated elevators and elevator deflections.

Actuator Failure. The left outboard elevator is stuck at -5 degrees after 1 second, that is,

$$
u_{1}(t)=-5 \mathrm{deg}, \quad \text { for } t \geq t_{f} \text { second, }
$$

where $t_{f}=1$ second. The signal $u_{1}(t)$ is the output of the left outboard elevator. For $t>t_{f}$ the elevator does not respond to the elevator input $v_{1}(t)$.
Simulation Results. The results are shown in Figures 15 and 16. From Figure 15, we can see that the plant state tracks the reference state after failure. Figure 16 shows the designed elevator signal, elevator inputs, and elevator outputs. The failure occurs at $t_{f}=1$ second and the other elevators can be seen to accommodate the failure with the adaptation.

3.3.2. NASA Generic Transport Model. Here we apply the adaptive control scheme to the nonlinear NASA GTM.

LTI Model for Controller Design. For adaptive control design, we consider a LTI model for a wings levelled flight having a 3 deg angle of attack at trim. Similar to the linear simulation study, we study the compensation for constant elevator failures and will consider multiple elevator failures in which both the lateral and longitudinal are active. The state and control vectors are given by

$$
\begin{gathered}
x=[u, q, \theta, p, r, \phi, \psi], \\
u=\left[\delta_{\text {elob }}, \delta_{\text {elib }}, \delta_{\text {erob }}, \delta_{\text {erib }}, \delta_{\mathrm{tl}}, \delta_{\mathrm{tr}}, \delta_{\mathrm{al}}, \delta_{\mathrm{ar}}, \delta_{\mathrm{ru}}, \delta_{\mathrm{rl}}\right],
\end{gathered}
$$

where $\delta_{\text {elob }}, \delta_{\text {elib }}, \delta_{\text {erob}}$, and $\delta_{\text {erib }}$ are the four elevator segments, $\delta_{\mathrm{tl}}$ and $\delta_{\mathrm{tr}}$ are the two engine throttles, $\delta_{\mathrm{al}}$ and $\delta_{\mathrm{ar}}$ are left and right aileron segments, and $\delta_{\mathrm{ru}}$ and $\delta_{\mathrm{rl}}$ are upper and lower rudder segments. The actuators will be grouped into elevator, engine, aileron, and rudder groups. A virtual control signal is designed for each group and allocated to its members adaptively. The design is based on that in Section 3.2 and is similar to that in the linear simulation study. We include lateral states and lateral actuators to 


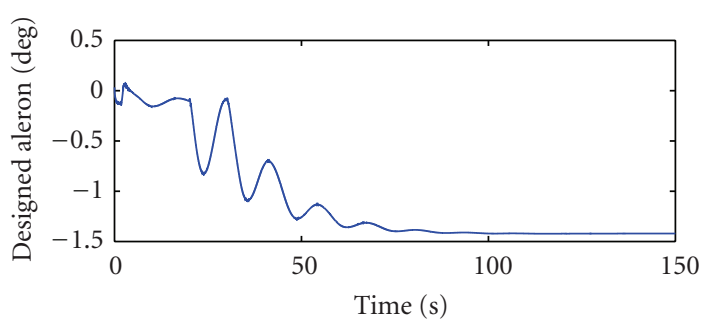

(a)

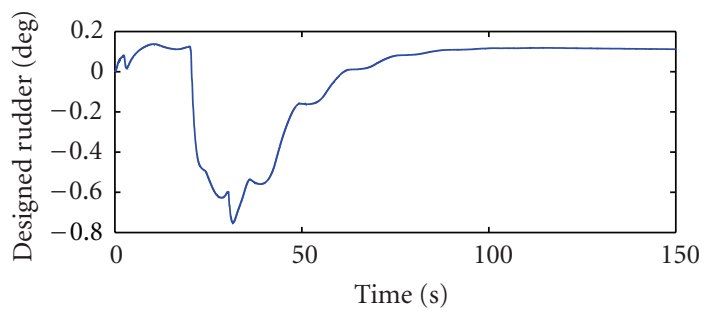

(c)

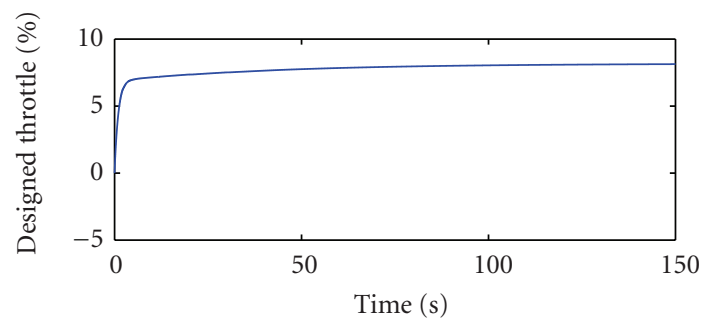

(e)

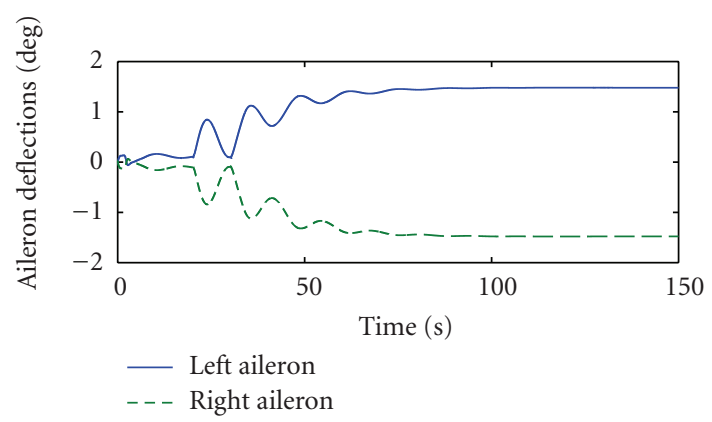

(b)

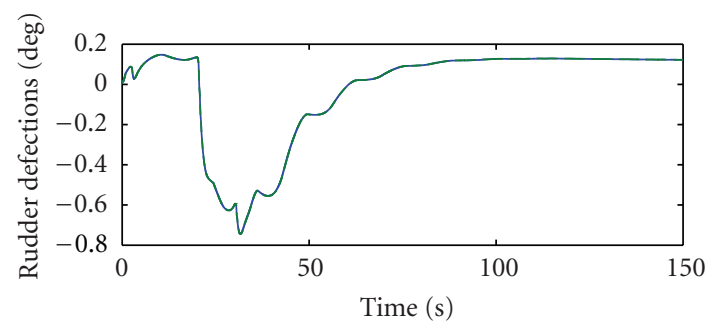

- Upper rudder

- - - Lower rudder

(d)

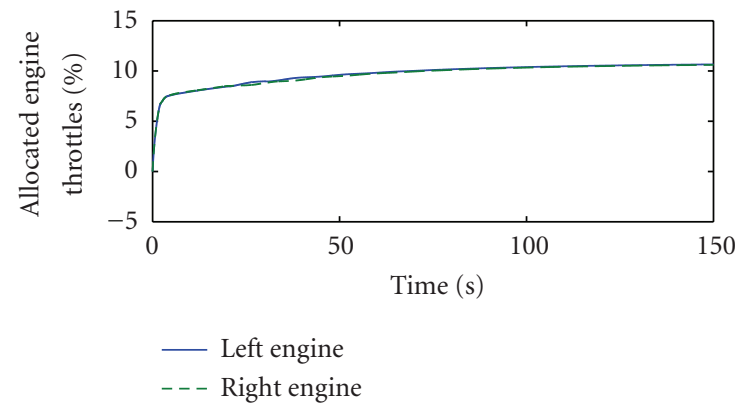

(f)

FIGURE 21: Time history of the inputs and outputs of ailerons, rudders, and engines.

regulate the disturbance of the elevator failures propagated to the lateral dynamics.

Flight Conditions. A set of commands that aim to make the aircraft climb at 4-degree pitch angle is applied at 5 seconds. The nominal parameters and reference inputs are obtained as in the linear simulation example.

Actuator Failures. The right outboard elevator is locked at 10 degrees at 10 seconds and the right inboard elevator at 5 degrees at 20 seconds. The control objective is to maintain the climbing flight in the presence of these failures.

The simulation results are shown in Figures 17-23. Figure 17 shows the time history of the longitudinal states and reference model states. It can be seen that the tracking of the desired longitudinal attitude is accurately achieved.

The lateral states are shown in Figure 18. Some disturbances can be observed after the occurrence of asymmetric elevator failures. These disturbances are regulated by the ailerons and asymptotic tracking of the lateral states can be also achieved.

Figure 19 shows the flight trajectory, ground track, and altitude of the aircraft. From the ground track plot, we can see the effect of asymmetric failure on the lateral dynamics. The deviation is corrected by the lateral actuators. Figure 20 shows the lumped elevator signal, allocated elevator signals, and the actual elevator deflections. The elevator failures appear as constant (step signals) on the lower plot, and the designed and allocated elevator signals are shown to accommodate for the failures after their occurrence. The input signals and outputs of other actuators are shown in Figure 21. Note the actuation of the aileron and rudder required to compensate for the asymmetric failure. The time history of the control allocation gains and some selected adaptive parameters are shown in Figures 22 and 23. It can be seen that the allocation gains and controller parameters are updated autonomously to ensure the desired flight. 


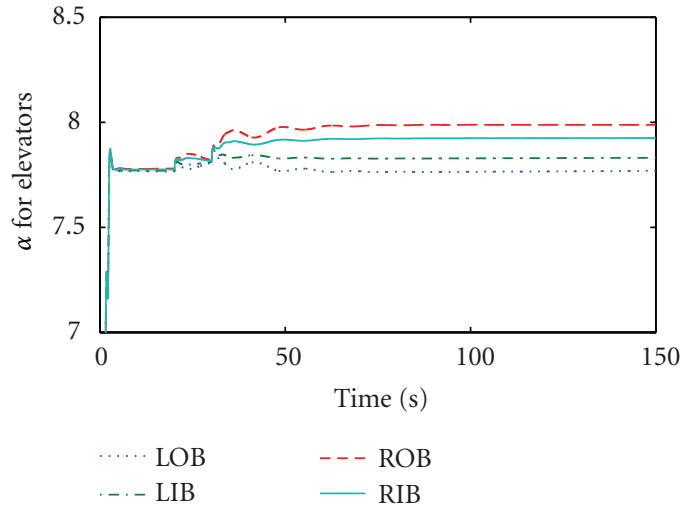

(a)

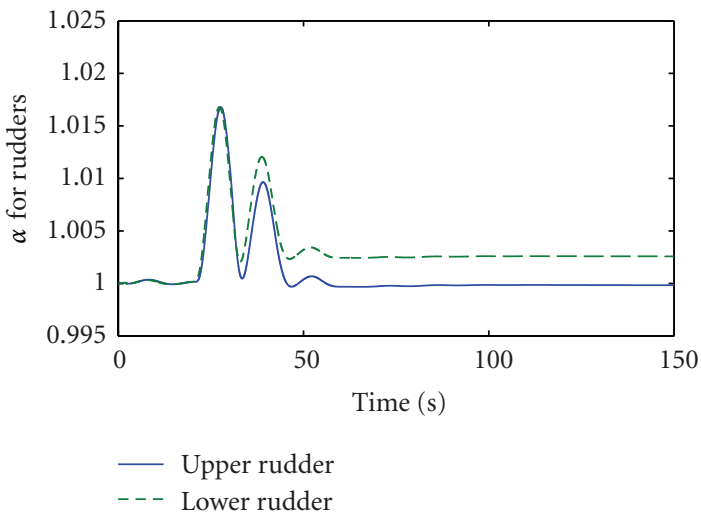

(c)

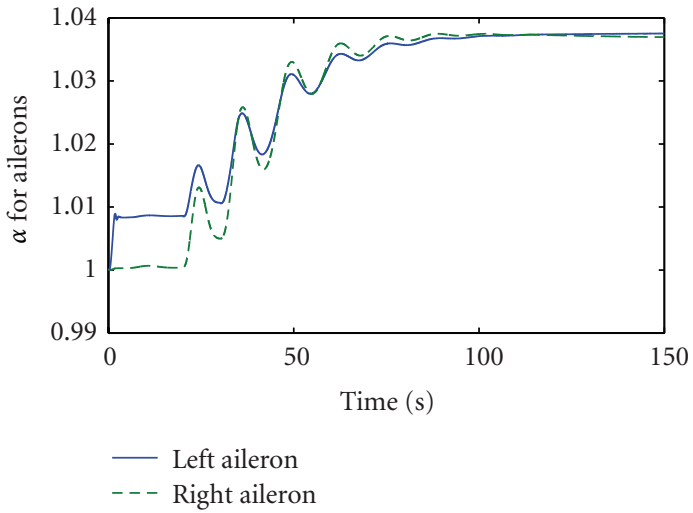

(b)

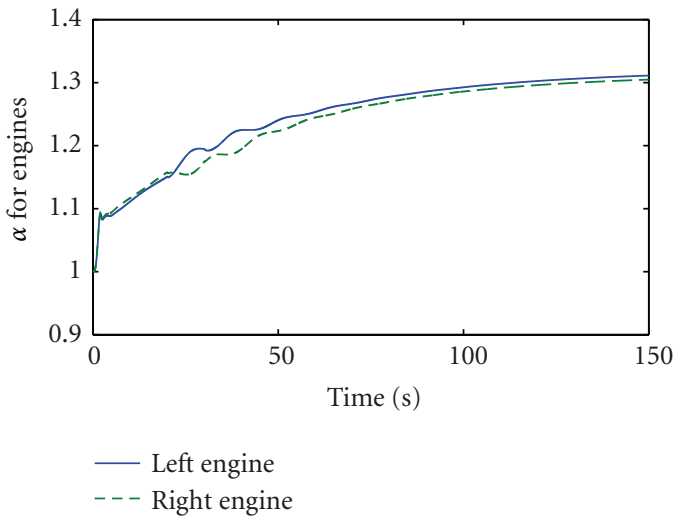

(d)

FIgURE 22: Time history of control allocation gains.

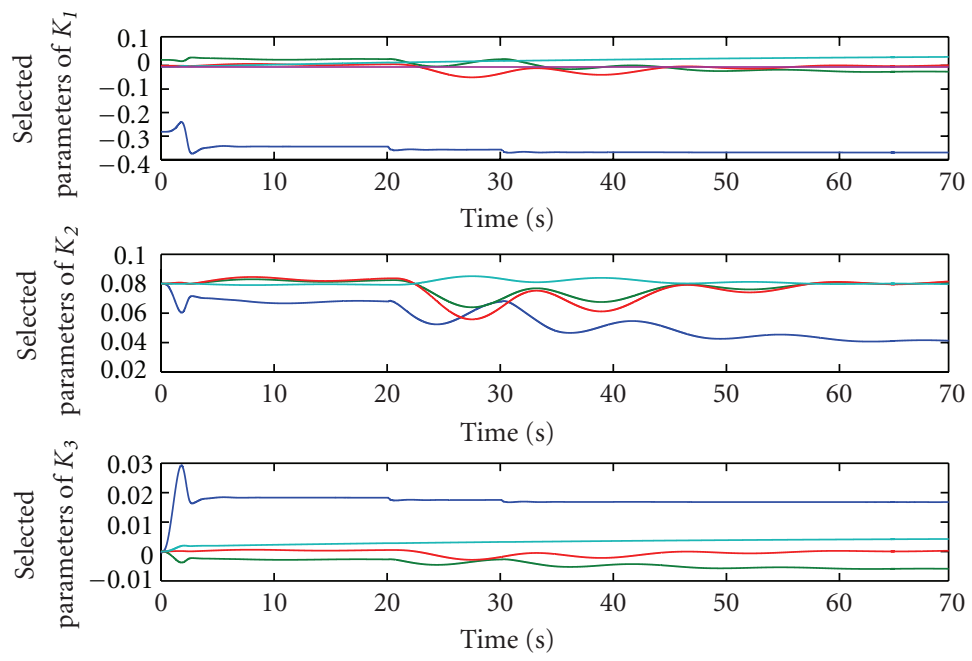

FIGURE 23: Time history of selected adaptive controller parameters.

\section{Conclusions}

A novel adaptive control allocation framework is proposed herein. The adaptive allocation scheme includes an adaptive control signal and a control allocation unit with adaptively updated allocation gains. Two adaptive control allocation algorithms have been proposed for the compensation of uncertain failures. The proposed algorithms have been shown to guarantee closed-loop stability and asymptotic state tracking. It has also been shown that the proposed adaptive control allocation framework reduces the controller complexity with proper grouping of the actuators. In this 
framework, the control signal to be adaptively allocated can be actuated by nonadaptive controllers. The simulation results demonstrate the performance of the proposed algorithms and their applicability to aircraft flight control. Some future research topics in this direction include the extension of the adaptive control allocation framework to systems with multiple groups of actuators and the strict enforcement of a pure allocation, that is, exactly enforcing the designed control signal with $\sum \alpha_{i}=1$.

\section{Appendix}

Proof of Theorem 1. When a failure occurs, the control effectiveness matrix $\Lambda$ has a discontinuity. It would lead to a finite jump in $\widetilde{\alpha}(t)$ as well, which could result in a a finite jump in the error dynamics. Let us assume that there are $l$ failures occurring in the system, and the actuator failures occur at time instants $t_{k}$, with $t_{k}<t_{k+1}, k=1,2, \ldots, N$. For the closed-loop stability and state tracking analysis, we choose the following Lyapunov-like function:

$$
V(t)=\frac{1}{2} e^{\top}(t) P e(t)+\frac{1}{2} \tilde{\alpha}^{\top}(t) \Gamma_{\alpha}^{-1} \Lambda^{-1} \widetilde{\alpha}(t)+\frac{1}{2} \tilde{\theta}^{\top}(t) \Gamma_{\theta}^{-1} \tilde{\theta}(t),
$$

for each time interval $\left(t_{k}, t_{k+1}\right), k=0,1, \ldots, N$ with $t_{0}=0$ and $t_{N+1}=\infty \cdot V(t)$ is thus discontinuous with finite jumps at $t_{k}, k=1, \ldots, N$. Taking the time derivative of $V(t)$ and substituting the adaptive laws in (11) and (12) into the result for each $\left(t_{k}, t_{k+1}\right)$, we obtain

$$
\begin{aligned}
\dot{V}(t)= & e^{\top}(t) P \dot{e}(t)+\tilde{\alpha}^{\top}(t) \Gamma_{\alpha}^{-1} \dot{\alpha}(t)+\tilde{\theta}^{\top}(t) \Gamma_{\theta}^{-1} \dot{\theta}(t) \\
= & e^{\top}(t) P\left[A_{m} e(t)+b_{0} \widetilde{\theta}^{\top}(t) \omega(t)+B \tilde{\alpha}(t) v_{0}(t)\right] \\
& -\tilde{\alpha}^{\top}(t) B^{\top} P e(t) v_{0}(t)-\tilde{\theta}^{\top}(t) \omega(t) e^{\top}(t) P b_{0} \\
= & -\frac{1}{2} e^{\top} Q e \leq 0 .
\end{aligned}
$$

Thus we can conclude that for each $\left(t_{k}, t_{k+1}\right), k=0,1, \ldots, N$, $V(t)$ is bounded. Since $V(t)$ only has finite jumps at $t_{k}$, $k=1, \ldots, N$, we can conclude that $V(t)$ is bounded for $t \in[0, \infty)$, and $e(t) \in L^{\infty}, \tilde{\alpha}(t) \in L^{\infty}, \tilde{\theta}(t) \in L^{\infty}, \alpha(\mathrm{t}) \in L^{\infty}$, $\theta(t) \in L^{\infty}, x(t) \in L^{\infty}$, and $\omega(t) \in L^{\infty}$. Integrating both sides of (A.2), we can obtain

$$
V\left(t_{k}^{+}\right)-V\left(t_{k+1}^{-}\right)=\frac{1}{2} \int_{t_{k}}^{t_{k+1}} e^{\top}(\tau) Q(\tau) e(\tau) d \tau
$$

For $N+1$ intervals: $\left[0, t_{1}\right),\left(t_{1}, t_{2}\right), \ldots,\left(t_{N-1}, t_{N}\right)$, and $\left(t_{N}, \infty\right)$, (A.3) holds. Summing both sides of (A.3) for $k=0,1, \ldots, N$, we obtain

$$
\begin{aligned}
\frac{1}{2} \int_{0}^{\infty} e^{\top}(\tau) Q(\tau) e(\tau) d \tau= & V(0)-V\left(t_{1}^{-}\right)+V\left(t_{1}^{+}\right) \\
& -V\left(t_{2}^{-}\right)+V\left(t_{2}^{+}\right)-\cdots-V\left(t_{k}^{-}\right) \\
& +V\left(t_{k}^{+}\right)-\cdots-V\left(t_{N}^{-}\right)+V\left(t_{N}^{+}\right) \\
& -V(\infty) \\
= & V(0)+\sum_{i=1}^{N}\left[V\left(t_{i}^{+}\right)-V\left(t_{i}^{-}\right)\right] \\
& -V(\infty)<\infty,
\end{aligned}
$$

because the jumps $V\left(t_{i}^{+}\right)-V\left(t_{i}^{-}\right)$are finite and the number $N$ of jumps is also finite. Thus we have $e(t) \in L^{2}$. We can also conclude from (10) that $\dot{e}(t) \in L^{\infty}$. So from $e(t) \in L^{2} \cap L^{\infty}$, and $\dot{e}(t) \in L^{\infty}$, we have $\lim _{0} \rightarrow \infty e(t)=0$.

Proof of Theorem 3. In Section 3.1, we have assumed that there are at most $p \leq m-1$ constant actuator failures and defined an index set for failed actuators as $\mathcal{F}=\left\{i_{1}, \ldots, i_{p}\right\}$ such that $\sigma_{k}=1$ for all $k \in \mathcal{F}$. Here we further assume that the failures occur at instants $t_{k}$, with $t_{k}<t_{k+1}, k=1,2, \ldots, N$ with $1 \leq N \leq p$. The number of failure instants may be smaller than the total number of failures since multiple failures may happen at the same time. For the stability proof, we choose the following Lyapunov-like function

$$
V(t)=\frac{1}{2} e^{\top}(t) P e(t)+\frac{1}{2} \sum_{i \notin \mathcal{F}} \gamma_{i}^{-1} \widetilde{\alpha}_{i}^{2}(t)+\frac{1}{2} \widetilde{\theta}^{\top}(t) \Gamma_{\theta}^{-1} \tilde{\theta}(t),
$$

for each time interval $\left(t_{k}, t_{k+1}\right), k=0,1, \ldots, N$, with $t_{0}=$ 0 and $t_{N+1}=\infty$. The time derivative in each time interval $\left(t_{k}, t_{k+1}\right)$ is

$$
\begin{aligned}
\dot{V}(t)= & e^{\top}(t) P \dot{e}(t)+\sum_{i \notin \mathcal{F}} \gamma_{i}^{-1} \widetilde{\alpha}_{i}(t) \dot{\alpha}_{i}(t)+\tilde{\theta}^{\top}(t) \Gamma_{\theta}^{-1} \dot{\bar{\theta}}(t) \\
= & e(t)^{\top} P A_{m} e(t)+e^{\top}(t) P B\left(I-\sigma_{f}\right) \tilde{\alpha}(t) v_{0}(t) \\
& +e^{\top}(t) P b_{0} \tilde{\theta}^{\top}(t) \bar{\omega}(t) \\
& -e^{\top}(t) P v_{0}(t) \sum_{i \notin \mathcal{F}} \tilde{\alpha}_{i}(t) b_{i}-\tilde{\theta}^{\top}(t) \bar{\omega}(t) e^{\top}(t) P b_{0} \\
= & e^{\top}(t) P A_{m} e(t)=-\frac{1}{2} e^{\top}(t) Q e(t) \leq 0
\end{aligned}
$$

with the fact that $B\left(I-\sigma_{f}\right) \widetilde{\alpha}(t)=\sum_{i \notin \mathcal{F}} \tilde{\alpha}_{i}(t) b_{i}$. Following a similar approach to the stability analysis in Section 2, we can conclude that for any $t \in[0, \infty), V(t) \in L^{\infty}, e(t) \in$ $L^{\infty}, x(t) \in L^{\infty}, \bar{\omega}(t) \in L^{\infty}, \bar{\theta}(t) \in L^{\infty}, v_{0}(t) \in L^{\infty}, e(t) \in$ $L^{2}$. Since $V(t)$ only includes $\widetilde{\alpha}_{i}(t)$ with $i \notin \mathcal{F}$, we can only conclude the boundedness of $\tilde{\alpha}_{i}(t)$ and $\alpha_{i}(t)$ for $i \notin \mathcal{F}$. To show the boundedness of $\alpha_{j}(t), j \in \mathcal{F}$, we note that for any $j \in \mathcal{F}$

$$
\alpha_{j}(t)=\alpha_{j}(0)-\int_{0}^{t} \gamma_{j} e^{\top}(\tau) P v_{0}(\tau) b_{j} d \tau
$$


based on the adaptive law in (42). Note that all the columns of $B$ are linearly dependent based on the rank condition in Assumption 2. So we can have

$$
b_{j}=c_{j}^{*} b_{k}, \quad \forall j \in \mathcal{F},
$$

where $b_{k}$ is the column of $B$ that corresponds to an arbitrary healthy actuator, that is, $k \notin \mathcal{F}$, and $c_{j}^{*}$ is a nonzero constant. Equation (A.7) can thus be expressed as

$$
\begin{aligned}
\alpha_{j}(t) & =\alpha_{j}(0)-\int_{0}^{t} \gamma_{j} e^{\top}(t) P v_{0}(t) c_{j}^{*} b_{k} d t \\
& =\alpha_{j}(0)-\frac{\gamma_{j}}{\gamma_{k}} c_{j}^{*} \int_{0}^{t} \gamma_{k} e^{\top}(t) P v_{0}(t) b_{k} d t \\
& =\alpha_{j}(0)+\frac{\gamma_{j}}{\gamma_{k}} c_{j}^{*} \int_{0}^{t}\left(-\gamma_{k} e^{\top}(t) P v_{0}(t) b_{k}\right) d t \\
& =\alpha_{j}(0)+\frac{\gamma_{j}}{\gamma_{k}} c_{j}^{*} \int_{0}^{t} \dot{\alpha}_{k}(t) d t \\
& =\alpha_{j}(0)+\frac{\gamma_{j}}{\gamma_{k}} c_{j}^{*}\left[\alpha_{k}(t)-\alpha_{k}(0)\right], \quad \forall j \in \mathcal{F} .
\end{aligned}
$$

Since $\alpha_{k}(t), k \notin \mathcal{F}$ has been proved to be bounded, we have $\alpha_{j}(t) \in L^{\infty}$, for $j \in \mathcal{F}$.

We can further obtain that $\dot{e}(t) \in L^{\infty}$ from (40). With $e(t) \in L^{\infty} \cap L^{2}$ and $\dot{e}(t) \in L^{\infty}$, we can have $\lim _{t \rightarrow \infty} e(t)=$ 0 .

\section{Acknowledgments}

This work was supported by the NRA NNX08AC62A of the IRAC project of NASA. The authors would like to thank Drs. Suresh M. Joshi and Sean P. Kenny at the NASA Langley Research Center for their valuable comments.

\section{References}

[1] M. W. Oppenheimer, D. B. Doman, and M. A. Bolender, "Control allocation for over-actuated systems," in Proceedings of the 14th Mediterranean Conference on Control and Automation (MED '06), pp. 1-6, June 2006.

[2] J. M. Buffington and D. F. Enns, "Lyapunov stability analysis of daisy chain control allocation," Journal of Guidance, Control, and Dynamics, vol. 19, no. 6, pp. 1226-1230, 1996.

[3] J. B. Davidson, F. J. Lallman, and W. T. Bundick, "Real-time adaptive control allocation applied to a high performance aircraft," in Proceedings of the 5th SIAM Conference on Control and Its Applications, 2001.

[4] J. D. Bošković and R. K. Mehra, "Control allocation in overactuated aircraft under position and rate limiting," in Proceedings of the American Control Conference, pp. 791-796, Anchorage, Alaska, USA, May 2002.

[5] Y. Luo, A. Serrani, S. Yurkovich, M. W. Oppenheimer, and D. B. Doman, "Model-predictive dynamic control allocation scheme for reentry vehicles," Journal of Guidance, Control, and Dynamics, vol. 30, no. 1, pp. 100-113, 2007.

[6] R. Venkataraman, M. Oppenheimer, and D. Doman, "A new control allocation method that accounts for effector dynamics," in Proceedings of the IEEE Aerospace Conference Proceedings, pp. 2710-2715, March 2004.
[7] F. Fahroo and D. Doman, "A direct method for approach and landing trajectory reshaping with failure effect estimation," in Proceedings of AIAA Guidance, Navigation, and Control Conference (AIAA '04), pp. 200-209, August 2004.

[8] M. A. Holender and D. B. Doman, "Nonlinear control allocation using piecewise linear functions," Journal of Guidance, Control, and Dynamics, vol. 27, no. 6, pp. 1017-1027, 2004.

[9] D. B. Doman, B. J. Gamble, and A. D. Ngo, "Quantized control allocation of reaction control jets and aerodynamic control surfaces," Journal of Guidance, Control, and Dynamics, vol. 32, no. 1, pp. 13-24, 2009.

[10] M. Marwaha and J. Valasek, "Fault-tolerant control allocation for Mars entry vehicle using adaptive control," International Journal of Adaptive Control and Signal Processing, vol. 25, no. 2, pp. 95-113, 2011.

[11] G. Tao, S. Chen, X. Tang, and S. M. Joshi, Adaptive Control of Systems with Actuator Failures, Springer, London, UK, 2004.

[12] L. G. Crespo, M. Matsutani, and A. M. Annaswamy, "Design of a model reference adaptive controller for a remotely operated air vehicle," AIAA Journal of Guidance, Control and Dynamics, vol. 35, no. 2, pp. 406-422, 2012.

[13] Y. Liu, X. Tang, G. Tao, and S. M. Joshi, "Adaptive compensation of aircraft actuation failures using an engine differential model," IEEE Transactions on Control Systems Technology, vol. 16, no. 5, pp. 971-982, 2008.

[14] G. Tao, Adaptive Control Design and Analysis, John Wiley \& Sons, 2003.

[15] X. Tang, Y. Liu, and G. Tao, "A study of adaptation of multiple actuating signals for LTI systems," in Proceedings of the American Control Conference, pp. 5996-6001, June 2006.

[16] T. Jodan, W. Langford, C. Belcastro et al., "Development of a dynamically scaled generic transport model testbed for flight research experiments," in Proceedings of the AUVSIs Unmanned Systems North America Symposium and Exhibition, August 2004.

[17] T. L. Jordan, W. M. Langford, and J. S. Hill, "Airborne-subscale transport aircraft research testbed-aircraft model development," in Proceedings of the AIAA Guidance, Navigation, and Control Conference, pp. 5767-5778, San Francisco, Calif, USA, August 2005. 

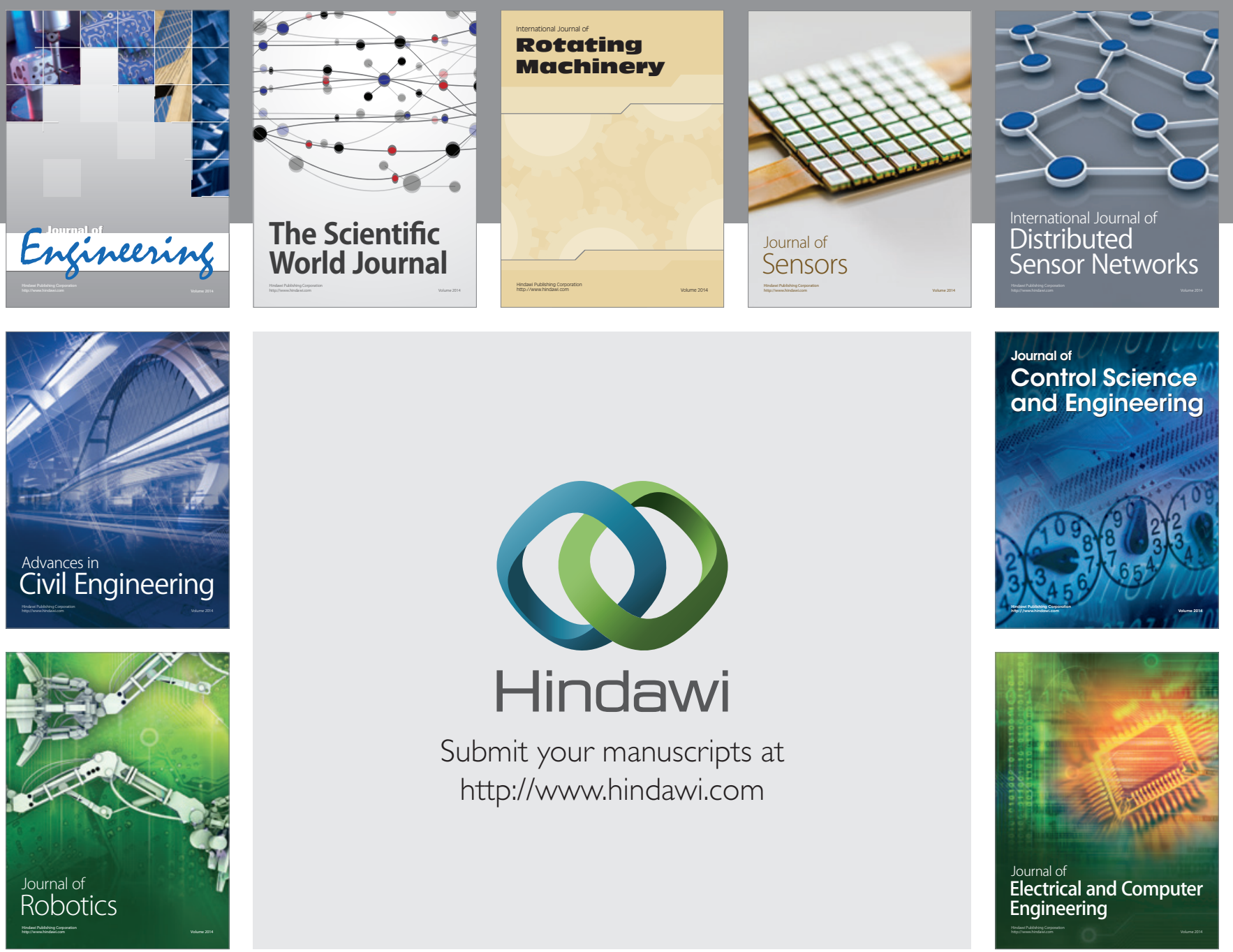

Submit your manuscripts at

http://www.hindawi.com
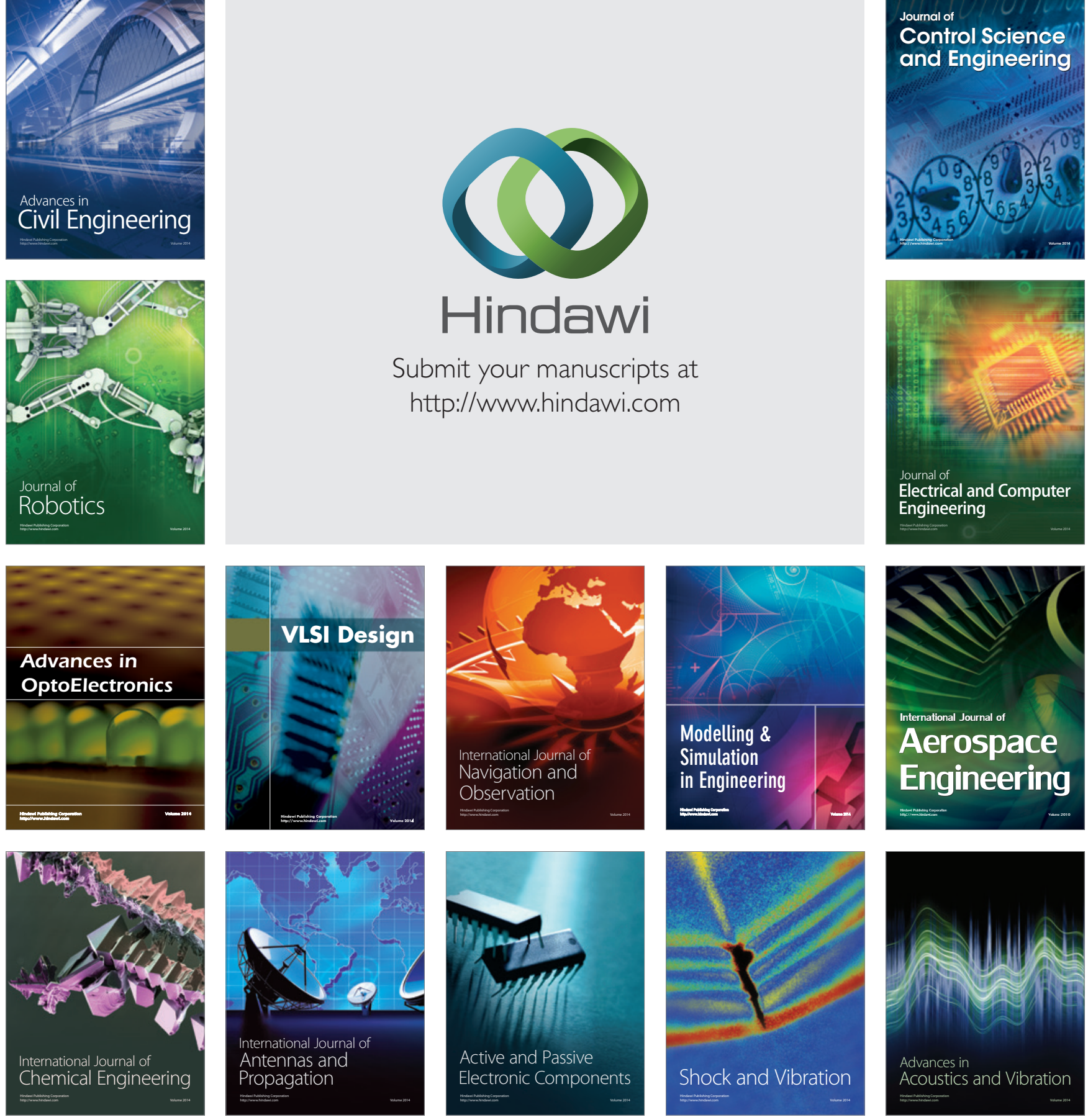